\title{
The $p$ th moment asymptotic stability and exponential stability of stochastic functional differential equations with polynomial growth condition
}

\author{
Lichao Feng ${ }^{1,2}$ and Shoumei Li ${ }^{1 *}$
}

"Correspondence:

lisma@bjut.edu.cn

${ }^{1}$ College of Applied Sciences,

Beijing University of Technology, 100 Pingleyuan, Chaoyang District, Beijing, 100124, P.R. China Full list of author information is available at the end of the article

\begin{abstract}
This paper mainly discusses the pth moment asymptotic stability and the exponential stability of nonlinear stochastic functional differential equations (SFDEs) satisfying the local Lipschitz condition but not the linear growth condition. These new conditions assume that the coefficients of SFDEs are polynomials or dominated by polynomials. We establish some sufficient conditions for the pth moment asymptotic stability and the exponential stability of nonlinear SFDEs by applying some novel techniques. Some nontrivial examples are provided to illustrate our results.

MSC: $93 \mathrm{E} 03 ;$ 93D20

Keywords: stochastic functional differential equations; polynomial growth condition; pth moment asymptotic stability; pth moment exponential stability; almost sure exponential stability
\end{abstract}

\section{Introduction}

Stochastic modeling plays an important role in describing the dynamical systems in many branches of science and industries. Since the introduction of Itô's formula, stochastic functional differential equations (SFDEs) have been used successfully to model such systems in many application fields such as biology, engineering, economics and finance (e.g., [1, 2]). The stability of SFDEs, including stochastic delay differential equations (SDDEs), is the most fundamental problem. It has received lots of attention over past years, and many important results have been established by many researchers (e.g., [1, 3-13]). In particular, by exploiting the positivity of the solution, Appleby and Reynolds [14] studied the exponential and non-exponential convergence rates of linear convolution Itô-Volterra equations. Then Appleby and Rodkina [15] discussed the stochastic stability and stochastic asymptotic stability of a nonlinear Volterra difference equation. Mao [7] considered the exponential stability of linear SDDEs, and he also extended it to the case of semi-linear uncertain SDDEs [9]. Mao [8] investigated Razumikhin-type theorems on the exponential stability of SFDEs whose coefficients satisfy the local Lipschitz condition and the linear growth condition, which was used to study the exponential stability of stochastic interval systems [4].

○2014 Feng and Li; licensee Springer. This is an Open Access article distributed under the terms of the Creative Commons Attribution License (http://creativecommons.org/licenses/by/2.0), which permits unrestricted use, distribution, and reproduction in any medium, provided the original work is properly cited. 
On the one hand, most of the stability criteria concentrate on the exponential stability. In many practical uses, however, stabilizing a system exponentially fast is not economical and sometimes unfeasible. Therefore, it is also significant to study the asymptotic stability for SFDEs further. For example, Mao [10] obtained useful criteria on the almost sure asymptotic stability for SDDEs. By using Razumikhin technique, Huang and Deng [3] studied the general $p$ th moment asymptotic stability of SFDEs.

On the other hand, most of the stability criteria above require the coefficients of corresponding systems to satisfy the linear growth condition or the one-side linear growth condition. However, many stochastic systems do not satisfy these conditions. Therefore, it is necessary to study the stability for stochastic systems without the linear growth condition or the one-side linear growth condition. Recently, Shen et al. [16] used the LaSalle technique to study the almost sure asymptotic stability of SFDEs without satisfying the linear growth condition. Then Luo et al. [6] established new criteria on the asymptotic stability and the boundedness of SFDEs where the linear growth condition was no longer needed. Liu et al. [5] studied the asymptotic stability of nonlinear SDDEs with the polynomial growth condition.

If the coefficients of SFDEs are polynomials or dominated by polynomials, the existing methods cannot be used directly (details can be found in examples in Section 6). Thus, it is natural to ask the following questions: What happens if SFDEs obey the polynomial growth condition? Is there a unique global solution? If yes, under what conditions do SFDEs have the properties of the $p$ th moment asymptotic stability and the exponential stability? In the following sections, we shall answer these questions. Our aims are to establish some new criteria on the $p$ th moment asymptotic stability and the exponential stability of SFDEs with the polynomial growth condition and the local Lipschitz condition.

The organization of this paper is as follows. In Section 2, we give some necessary notations and lemmas. In Section 3, we discuss the existence of the global solution and the $p$ th moment asymptotic stability of SFDEs. We find that the existence result depends only on constants $\kappa$ and $\bar{\kappa}$ but not on other constants. In Section 4, we give sufficient conditions for the $p$ th moment exponential stability and the almost sure exponential stability of SFDEs. To make our theory more applicable, Section 5 discusses generalized theory on the asymptotic stability of SFDEs. To show the applications of our results, some illustrative examples are given in the final section.

\section{Preliminaries}

Throughout this paper, unless otherwise specified, let $\left(\Omega, \mathscr{F},\left\{\mathscr{F}_{t}\right\}_{t \geq 0}, \mathbb{P}\right)$ be a complete probability space with a filtration $\left\{\mathscr{F}_{t}\right\}_{t \geq 0}$ satisfying the usual conditions, and let $B(t)$ be an $m$-dimensional Brownian motion defined on the probability space. Let $\tau>0$ and $C\left([-\tau, 0] ; R^{n}\right)$ denote the family of all continuous $R^{n}$-valued functions $\varphi$ on $[-\tau, 0]$ with the norm $\|\varphi\|=\sup _{-\tau \leq \theta \leq 0}|\varphi(\theta)|$. Let $C=C_{\mathscr{F}_{0}}^{b}\left([-\tau, 0] ; R^{n}\right)$ be the family of all bounded, $\mathscr{F}_{0}$-measurable, $C\left([-\tau, 0] ; R^{n}\right)$-valued, $\mathscr{F}_{t}$-adapted stochastic processes. Let $\eta_{i}$ be probability measures on $[-\tau, 0]$ which satisfy $\int_{-\tau}^{0} \mathrm{~d} \eta_{i}(\theta)=1(i=1,2,3,4)$. Let $L^{1}\left(R_{+} ; R_{+}\right)$be the family of all functions $\xi: R_{+} \rightarrow R_{+}$such that $\int_{0}^{+\infty} \xi(t) \mathrm{d} t<\infty$. We assume that $\mathbf{x}(t)$ is a continuous $R$-valued stochastic process on $t \in[-\tau, \infty), \mathbf{x}_{t}=\{\mathbf{x}(t+\theta):-\tau \leq \theta \leq 0\}$ for all $t \geq 0$, which is regarded as a $C\left([-\tau, 0] ; R^{n}\right)$-valued stochastic process.

Consider an $n$-dimensional SFDE

$$
\mathrm{d} \mathbf{x}(t)=\mathbf{f}\left(\mathbf{x}_{t}, t\right) \mathrm{d} t+\mathbf{g}\left(\mathbf{x}_{t}, t\right) \mathrm{d} B(t)
$$


on $t \geq 0$ with initial data $\{\mathbf{x}(\theta):-\tau \leq \theta \leq 0\}=\zeta \in C_{\mathscr{F}_{0}}^{b}\left([-\tau, 0] ; R^{n}\right)$, where

$$
\mathbf{f}: C\left([-\tau, 0] ; R^{n}\right) \times R_{+} \rightarrow R^{n}, \quad \mathbf{g}: C\left([-\tau, 0] ; R^{n}\right) \times R_{+} \rightarrow R^{n \times m}
$$

Assume, furthermore, that $\mathbf{f}(0, t)=0$ and $\mathbf{g}(0, t)=0$, so system (1) has the solution $\mathbf{x}(t)=0$. The solution is called a trial solution or an equilibrium solution.

It is said that $\mathbf{f}, \mathbf{g}$ satisfy the following local Lipschitz condition.

Assumption 2.1 For each integer $k \geq 1$, there is a positive $d_{k}$ such that

$$
|\mathbf{f}(\varphi, t)-\mathbf{f}(\psi, t)| \vee|\mathbf{g}(\varphi, t)-\mathbf{g}(\psi, t)| \leq d_{k}\|\boldsymbol{\varphi}-\psi\|
$$

for all $\varphi, \psi \in C\left([-\tau, 0] ; R^{n}\right), t \in R_{+}$with $\|\varphi\| \vee\|\psi\| \leq k$.

Replacing the linear growth condition or the one-side linear growth condition, we impose the following polynomial growth condition.

Assumption 2.2 There exist constants $\kappa, \bar{\kappa}, \hat{\kappa}, \underline{\kappa}, \gamma, \bar{\gamma}, \hat{\gamma}, \gamma \geq 0$, probability measures $\eta_{i}$ on $[-\tau, 0], i=1,2,3,4$, and positive numbers $n_{1}>1, n_{2}>1$ satisfying $n_{1}+1>2 n_{2}$, and bounded functions $\xi_{1}(t), \xi_{2}(t) \in L^{1}\left(R_{+} ; R_{+}\right)$such that

$$
\begin{aligned}
& \boldsymbol{\varphi}(0)^{T} \mathbf{f}(\boldsymbol{\varphi}, t) \leq-\kappa|\boldsymbol{\varphi}(0)|^{n_{1}+1}+\bar{\kappa} \int_{-\tau}^{0}|\boldsymbol{\varphi}(\theta)|^{n_{1}+1} \mathrm{~d} \eta_{1}(\theta)-\hat{\kappa}|\boldsymbol{\varphi}(0)|^{2} \\
&+\underline{\kappa} \int_{-\tau}^{0}|\boldsymbol{\varphi}(\theta)|^{2} \mathrm{~d} \eta_{2}(\theta)+\xi_{1}(t), \\
&|\mathbf{g}(\boldsymbol{\varphi}, t)| \leq \gamma|\boldsymbol{\varphi}(0)|^{n_{2}}+\bar{\gamma} \int_{-\tau}^{0}|\boldsymbol{\varphi}(\theta)|^{n_{2}} \mathrm{~d} \eta_{3}(\theta)+\hat{\gamma}|\boldsymbol{\varphi}(0)|+\underline{\gamma} \int_{-\tau}^{0}|\boldsymbol{\varphi}(\theta)| \mathrm{d} \eta_{4}(\theta)+\xi_{2}(t)
\end{aligned}
$$

for all $\varphi \in C\left([-\tau, 0] ; R^{n}\right), t \in R_{+}$.

Remark 1 In this paper, the probability measures $\eta_{i}, i=1,2,3,4$, can be weakened to any right-continuous nondecreasing functions (see [17]). Compared with [5], Assumption 2.2 in this paper is a generalization of the case of hypothesis $(\mathrm{H} 2)$ of [5].

Let $C^{2,1}\left(R^{n} \times[-\tau,+\infty) ; R_{+}\right)$denote the family of all continuous nonnegative functions $V(x, t)$ on $R^{n} \times[-\tau,+\infty)$ which are continuously twice differentiable in $x$ and once differentiable in $t$. For each $V \in C^{2,1}\left(R^{n} \times[-\tau,+\infty) ; R_{+}\right)$, denote an operator $\mathscr{L} V$ from $C\left([-\tau, 0] ; R^{n}\right) \times R_{+}$to $R$ by

$$
\mathscr{L} V(\varphi, t)=V_{t}(\varphi(0), t)+V_{x}(\varphi(0), t) \mathbf{f}(\varphi, t)+\frac{1}{2} \operatorname{trace}\left[\mathbf{g}^{T}(\varphi, t) V_{x x}(\varphi(0), t) \mathbf{g}(\varphi, t)\right]
$$

where

$$
\begin{aligned}
& V_{t}(\mathbf{x}, t)=\frac{\partial V(\mathbf{x}, t)}{\partial t}, \quad V_{x x}(\mathbf{x}, t)=\left(\frac{\partial^{2} V(\mathbf{x}, t)}{\partial x_{i} x_{j}}\right)_{n \times n}, \\
& V_{x}(\mathbf{x}, t)=\left(\frac{\partial V(\mathbf{x}, t)}{\partial x_{1}}, \ldots, \frac{\partial V(\mathbf{x}, t)}{\partial x_{n}}\right) .
\end{aligned}
$$

To obtain our main theorems, we recall a number of lemmas. 
Lemma 2.1 (Barbalat lemma [18]) If $h(t)$ is a uniformly continuous function on $[0, \infty)$, and $h(t) \in L^{1}\left(R_{+} ; R_{+}\right)$, then $\lim _{t \rightarrow \infty} h(t)=0$.

Lemma 2.2 (cf. [18]) If $h(t)$ is a bounded function on $[0, \infty)$, and $h(t) \in L^{1}\left(R_{+} ; R_{+}\right)$, then for any $\beta \geq 1, \int_{0}^{+\infty} h^{\beta}(t) \mathrm{d} t<\infty$.

Lemma 2.3 (cf. [5]) Assume $a, b, q>0, b \geq q, \alpha>\beta>0$. If the following condition holds,

$$
\frac{a}{b}>(\alpha-\beta) \beta^{\frac{\beta}{\alpha-\beta}} \alpha^{\frac{-\alpha}{\alpha-\beta}},
$$

then there exists $\bar{a} \in(0, a)$ satisfying, for all $t \geq 0$,

$$
a+b t^{\alpha}-q t^{\beta}>\bar{a} .
$$

Lemma 2.4 (cf. [12]) Assume $\alpha, \beta>0$. For any $h(t) \in C\left(R^{n} ; R\right)$, if $\lim \sup _{|t| \rightarrow \infty}\left(h(t) /|t|^{\alpha}\right)=0$, then there exists a constant $H$ satisfying

$$
\sup _{t \in R^{n}}\left\{-\beta|t|^{\alpha}+h(t)\right\}<H .
$$

Lemma 2.5 Let $A_{1}(t)$ and $A_{2}(t)$ be two continuous adapted increasing processes on $t \geq 0$ with $A_{1}(0)=A_{2}(0)=0$ a.s., let $M(t)$ be a real-valued continuous local martingale with $M(0)=0$ a.s., and let $\zeta$ be a nonnegative $\mathscr{F}_{0}$-measurable random variable such that $E \zeta<\infty$. Define $X(t)=\zeta+A_{1}(t)-A_{2}(t)+M(t)$ for $t \geq 0$. If $X(t)$ is nonnegative, then

$$
\left\{\lim _{t \rightarrow \infty} A_{1}(t)<\infty\right\} \subset\left\{\lim _{t \rightarrow \infty} X(t)<\infty\right\} \cap\left\{\lim _{t \rightarrow \infty} A_{2}(t)<\infty\right\} \quad \text { a.s. }
$$

where $C \subset D$ a.s. means $\mathbb{P}\left(C \cap D^{c}\right)=0$. In particular, if $\lim _{t \rightarrow \infty} A_{1}(t)<\infty$ a.s., then with probability one,

$$
\lim _{t \rightarrow \infty} X(t)<\infty, \quad \lim _{t \rightarrow \infty} A_{2}(t)<\infty, \quad-\infty<\lim _{t \rightarrow \infty} M(t)<\infty .
$$

This lemma is called nonnegative semi-martingale convergence theorem (see [19]), which will play an important role in this paper.

\section{3 pth moment asymptotic stability of SFDEs}

The classical theory is not used directly to system (1), so it is necessary to establish the following existence-and-uniqueness result.

Lemma 3.1 If Assumptions 2.1, 2.2 and $\kappa>\bar{\kappa}$ hold, then for any initial data $\zeta \in C$, there is a unique global solution $\mathbf{x}(t, \zeta)$ of system (1) on $t \geq-\tau$.

Remark 2 To ensure that a stochastic differential equation has a unique global solution for any given initial data, the coefficients of this equation are generally required to satisfy the linear growth condition and the local Lipschitz condition (see $[1,2,20])$ or the linear growth condition and some non-Lipschitz condition (see [21]), which shows that the linear growth condition plays an important role. In this paper, however, we only require the 
coefficients to satisfy the polynomial growth condition and the local Lipschitz condition. The solution of system (1) may explode at a finite time, so it is important to examine the existence and uniqueness of the global solution of system (1). Since it is not so easy to see this fact directly, we give the proof in the appendix. The fact that we write down our Lemma 3.1 here is to keep our paper completely based on Assumptions 2.1 and 2.2.

Compared with the non-explosion of the global solution (Lemma 3.1), the asymptotic boundedness in the sense of $p$ th moment is more interesting. We also state the following result of asymptotic boundedness and give the proof in the Appendix.

Lemma 3.2 If Assumptions 2.1, 2.2 and $\kappa>\bar{\kappa}$ hold, then for any $p \geq 0$ and any initial data $\zeta \in C$, there exists a constant $M_{p}>0$ such that the global solution $\mathbf{x}(t, \zeta)$ of system (1) has the property

$$
\sup _{-\tau \leq t<+\infty} E|\mathbf{x}(t, \zeta)|^{p} \leq M_{p}
$$

To study the $p$ th moment asymptotic stability, we prove the following lemma.

Lemma 3.3 If Assumptions 2.1, 2.2 and $\kappa>\bar{\kappa}$ hold, then for any $p \geq 2$ and any initial data $\zeta \in C, E|\mathbf{x}(t, \zeta)|^{p}$ is uniformly continuous on $[0, \infty)$, where $\mathbf{x}(t, \zeta)$ is the global solution of system (1).

Proof For the sake of simplicity, write $\mathbf{x}(t)=\mathbf{x}(t, \zeta), \mathbf{x}_{t}=\mathbf{x}_{t}^{\zeta}$. From Lemma 3.2, we know that for any $p \geq 2$, $\sup _{-\tau \leq t<\infty} E|\mathbf{x}(t)|^{p} \leq M_{p}$. Using Itô’s formula, we compute that, for any $0<s<t<\infty$,

$$
\begin{aligned}
\left.|E| \mathbf{x}(t)\right|^{p}-E|\mathbf{x}(s)|^{p} \mid \\
=\left.\frac{p}{2}\left|E \int_{s}^{t}\right| \mathbf{x}(r)\right|^{p-2}\left(2 \mathbf{x}^{T}(r) \mathbf{f}\left(\mathbf{x}_{r}, r\right)+\left|\mathbf{g}\left(\mathbf{x}_{r}, r\right)\right|^{2}+(p-2)|\mathbf{x}(r)|^{-2}\right. \\
\left.\quad \times\left|\mathbf{x}^{T}(r) \mathbf{g}\left(\mathbf{x}_{r}, r\right)\right|^{2}\right) \mathrm{d} r \mid \\
\leq \frac{p}{2} E \int_{s}^{t}\left[|\mathbf{x}(r)|^{p-2}\left(\left|2 \mathbf{x}^{T}(r) \mathbf{f}\left(\mathbf{x}_{r}, r\right)\right|+(p-1)\left|\mathbf{g}\left(\mathbf{x}_{r}, r\right)\right|^{2}\right)\right] \mathrm{d} r \\
\leq \frac{p}{2} \int_{s}^{t} E\left(2|\mathbf{x}(r)|^{p-2}\left|\mathbf{x}^{T}(r) \mathbf{f}\left(\mathbf{x}_{r}, r\right)\right|+(p-1)|\mathbf{x}(r)|^{p-2}\left|\mathbf{g}\left(\mathbf{x}_{r}, r\right)\right|^{2}\right) \mathrm{d} r .
\end{aligned}
$$

From Assumption 2.2, through simple computation we get that

$$
\begin{aligned}
\mid \mathbf{x}(r) & \left.\right|^{p-2}\left|\mathbf{x}^{T}(r) \mathbf{f}\left(\mathbf{x}_{r}, r\right)\right| \\
\leq & \frac{p-2}{p-1}|\mathbf{x}(r)|^{p-1}+\frac{1}{p-1}\left|\mathbf{x}^{T}(r) \mathbf{f}\left(\mathbf{x}_{r}, r\right)\right|^{p-1} \\
\leq & \frac{p-2}{p-1}|\mathbf{x}(r)|^{p-1}+\frac{5^{p-2}}{p-1}\left[\kappa^{p-1}|\mathbf{x}(r)|^{\left(n_{1}+1\right)(p-1)}\right. \\
& \quad+\bar{\kappa}^{p-1}\left(\int_{-\tau}^{0}|\mathbf{x}(r+\theta)|^{\left(n_{1}+1\right)} \mathrm{d} \eta_{1}(\theta)\right)^{p-1}+\hat{\kappa}^{p-1}|\mathbf{x}(r)|^{2(p-1)}
\end{aligned}
$$




$$
\begin{aligned}
& \left.+\underline{\kappa}^{p-1}\left(\int_{-\tau}^{0}|\mathbf{x}(r+\theta)|^{2} \mathrm{~d} \eta_{2}(\theta)\right)^{p-1}+\xi_{1}^{p-1}(r)\right], \\
|\mathbf{x}(r)|^{p-2}\left|\mathbf{g}\left(\mathbf{x}_{r}, r\right)\right|^{2} & \\
\leq & \frac{p-2}{p}|\mathbf{x}(r)|^{p}+\frac{2}{p}\left|\mathbf{g}\left(\mathbf{x}_{r}, r\right)\right|^{p} \\
\leq & \frac{p-2}{p}|\mathbf{x}(r)|^{p}+\frac{2}{p} 5^{p-1}\left[\gamma^{p}|\mathbf{x}(r)|^{p n_{2}}+\bar{\gamma}^{p}\left(\int_{-\tau}^{0}|\mathbf{x}(r+\theta)|^{n_{2}} \mathrm{~d} \eta_{3}(\theta)\right)^{p}\right. \\
& \left.+\hat{\gamma}^{p}|\mathbf{x}(r)|^{p}+\underline{\gamma}^{p}\left(\int_{-\tau}^{0}|\mathbf{x}(r+\theta)| \mathrm{d} \eta_{4}(\theta)\right)^{p}+\xi_{2}^{p}(r)\right],
\end{aligned}
$$

where we have used the elemental inequalities: for any $c, b, \alpha, \beta \geq 0, \alpha+\beta>0, c^{\alpha} b^{\beta} \leq$ $\frac{\alpha}{\alpha+\beta} c^{\alpha+\beta}+\frac{\beta}{\alpha+\beta} b^{\alpha+\beta}$; for any $c_{i} \geq 0, i=1,2, \ldots, d, \theta \geq 1,\left(\sum_{i=1}^{d} c_{i}\right)^{\theta} \leq d^{\theta-1} \sum_{i=1}^{d} c_{i}^{\theta}$.

By virtue of the boundedness of $\xi_{1}(t), \xi_{2}(t)$, assuming $\xi_{1}(t) \vee \xi_{2}(t) \leq \Psi$ and substituting (3) into (2), we have

$$
\begin{aligned}
\left.|E| \mathbf{x}(t)\right|^{p}-E|\mathbf{x}(s)|^{p} \mid & \\
\leq & \frac{p}{2} \int_{s}^{t} 2 \frac{p-2}{p-1} E|\mathbf{x}(r)|^{p-1}+2 \frac{5^{p-2}}{p-1}\left[\kappa^{p-1} E|\mathbf{x}(r)|^{\left(n_{1}+1\right)(p-1)}+\hat{\kappa}^{p-1} E|\mathbf{x}(r)|^{2(p-1)}\right. \\
& +\bar{\kappa}^{p-1} E\left(\int_{-\tau}^{0}|\mathbf{x}(r+\theta)|^{\left(n_{1}+1\right)} \mathrm{d} \eta_{1}(\theta)\right)^{p-1}+\underline{\kappa}^{p-1} E\left(\int_{-\tau}^{0}|\mathbf{x}(r+\theta)|^{2} \mathrm{~d} \eta_{2}(\theta)\right)^{p-1} \\
& \left.+\xi_{1}^{p-1}(r)\right]+\frac{p-2}{p}(p-1) E|\mathbf{x}(r)|^{p}+\frac{2}{p} 5^{p-1}(p-1)\left[\bar{\gamma}^{p} E\left(\int_{-\tau}^{0}|\mathbf{x}(r+\theta)|^{n_{2}} \mathrm{~d} \eta_{3}(\theta)\right)^{p}\right. \\
& \left.+\gamma^{p} E|\mathbf{x}(r)|^{p n_{2}}+\hat{\gamma}^{p} E|\mathbf{x}(r)|^{p}+\underline{\gamma}^{p} E\left(\int_{-\tau}^{0}|\mathbf{x}(r+\theta)| \mathrm{d} \eta_{4}(\theta)\right)^{p}+\xi_{2}^{p}(r)\right] \mathrm{d} r \\
\leq & \frac{p}{2} \int_{s}^{t} 2 \frac{p-2}{p-1} E|\mathbf{x}(r)|^{p-1}+2 \frac{5^{p-2}}{p-1}\left[\kappa^{p-1} E|\mathbf{x}(r)|^{\left(n_{1}+1\right)(p-1)}+\hat{\kappa}^{p-1} E|\mathbf{x}(r)|^{2(p-1)}\right. \\
& +\bar{\kappa}^{p-1} \int_{-\tau}^{0} E|\mathbf{x}(r+\theta)|^{\left(n_{1}+1\right)(p-1)} \mathrm{d} \eta_{1}(\theta)+\underline{\kappa}^{p-1} \int_{-\tau}^{0} E|\mathbf{x}(r+\theta)|^{2(p-1)} \mathrm{d} \eta_{2}(\theta) \\
& \left.+\xi_{1}^{p-1}(r)\right]+\frac{p-2}{p}(p-1) E|\mathbf{x}(r)|^{p}+\frac{2}{p} 5^{p-1}(p-1)\left[\bar{\gamma}^{p} \int_{-\tau}^{0} E|\mathbf{x}(r+\theta)|^{p n_{2}} \mathrm{~d} \eta_{3}(\theta)\right. \\
& \left.+\gamma^{p} E|\mathbf{x}(r)|^{p n_{2}}+\hat{\gamma}^{p} E|\mathbf{x}(r)|^{p}+\underline{\gamma}^{p} \int_{-\tau}^{0} E|\mathbf{x}(r+\theta)|^{p} \mathrm{~d} \eta_{4}(\theta)+\xi_{2}^{p}(r)\right] \mathrm{d} r \\
\leq & \frac{p}{2}\left[2 \frac{p-2}{p-1} M_{p-1}+2 \frac{5^{p-2}}{p-1}\left(\kappa^{p-1} M_{\left(n_{1}+1\right)(p-1)}+\bar{\kappa}^{p-1} M_{\left(n_{1}+1\right)(p-1)}\right.\right. \\
& \left.+\hat{\kappa}^{p-1} M_{2(p-1)}+\underline{\kappa}{ }^{p-1} M_{2(p-1)}+\Psi^{p-1}\right)+\frac{p-2}{p}(p-1) M_{p}+\frac{2}{p} 5^{p-1}(p-1) \\
& \left.\times\left(\gamma^{p} M_{p n_{2}}+\bar{\gamma}^{p} M_{p n_{2}}+\hat{\gamma}^{p} M_{p}+\underline{\gamma}^{p} M_{p}+\Psi^{p}\right)\right](t-s), \\
&
\end{aligned}
$$

which completes the proof of the uniform continuity of $E|\mathbf{x}(t)|^{p}$.

In this section, our aim is to study the $p$ th moment asymptotic stability of system (1). The following theorem establishes a new sufficient condition for the $p$ th moment asymptotic stability. 
Theorem 3.1 If Assumptions 2.1, 2.2 and the following condition (4) hold,

$$
\frac{(\gamma+\bar{\gamma})^{2}}{2(\kappa-\bar{\kappa})}+\frac{(\hat{\gamma}+\underline{\gamma})^{2}}{2(\hat{\kappa}-\underline{\kappa})-2 L(\kappa-\bar{\kappa})}<1, \quad(\hat{\kappa}-\underline{\kappa})-L(\kappa-\bar{\kappa})>0, \quad \kappa-\bar{\kappa}>0,
$$

then for any $p \in\left(0, p_{0}\right)$ and any initial data $\zeta \in C$, there is a unique global solution $\mathbf{x}(t, \zeta)$ of system (1) on $t \geq-\tau$, and $\mathbf{x}(t, \zeta)$ is pth moment asymptotically stable, namely

$$
\lim _{t \rightarrow \infty} E|\mathbf{x}(t, \zeta)|^{p}=0
$$

where $L=\left(n_{1}-2 n_{2}+1\right)\left(2 n_{2}-2\right)^{\frac{2 n_{2}-2}{n_{1}-2 n_{2}+1}}\left(n_{1}-1\right)^{\frac{1-n_{1}}{n_{1}-2 n_{2}+1}}, p_{0}=1+\left[\frac{(\gamma+\bar{\gamma})^{2}}{2(\kappa-\bar{\kappa})}+\frac{(\hat{\gamma}+\underline{\gamma})^{2}}{2(\hat{\kappa}-\underline{\kappa})-2 L(\kappa-\bar{\kappa})}\right]^{-1}$.

Proof From Lemma 3.1 and condition (4), there exists a unique global solution. For the sake of simplicity, write $\mathbf{x}(t)=\mathbf{x}(t, \zeta)$. Moreover, from the definition of $p_{0}$ and condition (4), we have $p_{0}>2$. For any $p \in\left[2, p_{0}\right)$, applying Itô's formula to $V(\mathbf{x}, t)=|\mathbf{x}(t)|^{p}$ and using the elemental inequalities (for any $c, b \in R, 0<\theta<1,(c+b)^{2} \leq \frac{c^{2}}{1-\theta}+\frac{b^{2}}{\theta}$ ), we obtain

$$
\begin{aligned}
& \mathscr{L} V \leq \frac{p}{2}|\mathbf{x}(t)|^{p-2}\left[-2 \kappa|\mathbf{x}(t)|^{n_{1}+1}+2 \bar{\kappa} \int_{-\tau}^{0}|\mathbf{x}(t+\theta)|^{n_{1}+1} \mathrm{~d} \eta_{1}(\theta)\right. \\
& -2 \hat{\kappa}|\mathbf{x}(t)|^{2}+2 \underline{\kappa} \int_{-\tau}^{0}|\mathbf{x}(t+\theta)|^{2} \mathrm{~d} \eta_{2}(\theta) \\
& +\frac{p-1}{\rho_{1}}\left(\frac{\gamma^{2}}{\delta_{1}}|\mathbf{x}(t)|^{2 n_{2}}+\frac{\bar{\gamma}^{2}}{1-\delta_{1}} \int_{-\tau}^{0}|\mathbf{x}(t+\theta)|^{2 n_{2}} \mathrm{~d} \eta_{3}(\theta)\right) \\
& +\frac{p-1}{\rho_{2}\left(1-\rho_{1}\right)}\left(\frac{\hat{\gamma}^{2}}{\delta_{2}}|\mathbf{x}(t)|^{2}+\frac{\underline{\gamma}^{2}}{1-\delta_{2}} \int_{-\tau}^{0}|\mathbf{x}(t+\theta)|^{2} \mathrm{~d} \eta_{4}(\theta)\right)+2 \xi_{1}(t) \\
& \left.+\frac{p-1}{\left(1-\rho_{1}\right)\left(1-\rho_{2}\right)} \xi_{2}^{2}(t)\right] \\
& \leq \frac{p}{2}\left[-2(\kappa-\bar{\kappa})|\mathbf{x}(t)|^{p+n_{1}-1}-\left(2 \hat{\kappa}-2 \underline{\kappa}-\frac{p-1}{\rho_{2}\left(1-\rho_{1}\right)}\left(\frac{\hat{\gamma}^{2}}{\delta_{2}}+\frac{\underline{\gamma}^{2}}{1-\delta_{2}}\right)-2 \frac{p-2}{p} \varepsilon_{1}^{p}\right.\right. \\
& \left.\left.-\frac{p-1}{1-\rho_{1}} \frac{1}{1-\rho_{2}} \frac{p-2}{p} \varepsilon_{2}^{p}\right)|\mathbf{x}(t)|^{p}+\frac{p-1}{\rho_{1}}\left(\frac{\gamma^{2}}{\delta_{1}}+\frac{\bar{\gamma}^{2}}{1-\delta_{1}}\right)|\mathbf{x}(t)|^{p+2 n_{2}-2}\right]+2 \underline{\kappa} J_{2} \\
& +\frac{p-1}{\rho_{2}\left(1-\rho_{1}\right)} \frac{\underline{\gamma}^{2}}{1-\delta_{2}} J_{4}+\frac{p}{2} \frac{p-1}{\rho_{1}} \frac{\bar{\gamma}^{2}}{1-\delta_{1}} \frac{2 n_{2}}{p+2 n_{2}-2} J_{3}+p \bar{\kappa} \frac{n_{1}+1}{p+n_{1}-1} J_{1} \\
& +2 \varepsilon_{1}^{-\frac{p(p-2)}{2}} \xi_{1}^{\frac{p}{2}}(t)+\frac{p-1}{1-\rho_{1}} \frac{1}{1-\rho_{2}} \varepsilon_{2}^{-\frac{p(p-2)}{2}} \xi_{2}^{p}(t),
\end{aligned}
$$

where $\rho_{1}, \rho_{2}, \delta_{1}, \delta_{2} \in(0,1), J_{1}=\int_{-\tau}^{0}|\mathbf{x}(t+\theta)|^{p+n_{1}-1} \mathrm{~d} \eta_{1}(\theta)-|\mathbf{x}(t)|^{p+n_{1}-1}, J_{2}=\int_{-\tau}^{0} \mid \mathbf{x}(t+$ $\theta)\left.\right|^{p} \mathrm{~d} \eta_{2}(\theta)-|\mathbf{x}(t)|^{p}, J_{3}=\int_{-\tau}^{0}|\mathbf{x}(t+\theta)|^{p+2 n_{2}-2} \mathrm{~d} \eta_{3}(\theta)-|\mathbf{x}(t)|^{p+2 n_{2}-2}, J_{4}=\int_{-\tau}^{0}|\mathbf{x}(t+\theta)|^{p} \mathrm{~d} \eta_{4}(\theta)-$ $|\mathbf{x}(t)|^{p}$. Setting $\delta_{1}=\frac{\gamma}{\gamma+\bar{\gamma}}, \delta_{2}=\frac{\hat{\gamma}}{\hat{\gamma}+\underline{\gamma}}$, we have

$$
\begin{aligned}
\mathscr{L} V \leq & \frac{p}{2}\left[-2(\kappa-\bar{\kappa})|\mathbf{x}(t)|^{p+n_{1}-1}+\frac{(p-1)(\gamma+\bar{\gamma})^{2}}{\rho_{1}}|\mathbf{x}(t)|^{p+2 n_{2}-2}-(2 \hat{\kappa}-2 \underline{\kappa}\right. \\
& \left.\left.-\frac{(p-1)(\hat{\gamma}+\underline{\gamma})^{2}}{\rho_{2}\left(1-\rho_{1}\right)}-2 \frac{p-2}{p} \varepsilon_{1}^{p}-\frac{p-1}{1-\rho_{1}} \frac{1}{1-\rho_{2}} \frac{p-2}{p} \varepsilon_{2}^{p}\right)|\mathbf{x}(t)|^{p}\right]+2 \underline{\kappa} J_{2}
\end{aligned}
$$




$$
\begin{aligned}
& +p \bar{\kappa} \frac{n_{1}+1}{p+n_{1}-1} J_{1}+\frac{p}{2} \frac{(p-1)(\bar{\gamma}+\gamma) \bar{\gamma}}{\rho_{1}} \frac{2 n_{2}}{p+2 n_{2}-2} J_{3}+\frac{(p-1)(\underline{\gamma}+\hat{\gamma}) \underline{\gamma}}{\rho_{2}\left(1-\rho_{1}\right)} J_{4} \\
& +2 \varepsilon_{1}^{-\frac{p(p-2)}{2}} \xi_{1}^{\frac{p}{2}}(t)+\frac{p-1}{1-\rho_{1}} \frac{1}{1-\rho_{2}} \varepsilon_{2}^{-\frac{p(p-2)}{2}} \xi_{2}^{p}(t) .
\end{aligned}
$$

Let $G(|\mathbf{x}(t)|)=\left(2(\hat{\kappa}-\underline{\kappa})-\frac{(\hat{\gamma}+\underline{\gamma})^{2}}{1-\rho_{1}}(p-1)\right)+2(\kappa-\bar{\kappa})|\mathbf{x}(t)|^{n_{1}-1}-\frac{p-1}{\rho_{1}}(\gamma+\bar{\gamma})^{2}|\mathbf{x}(t)|^{2 n_{2}-2}$. Because $p \in\left[2, p_{0}\right)$, then $(p-1) \frac{(\gamma+\bar{\gamma})^{2}}{2(\kappa-\bar{\kappa})}+(p-1) \frac{(\hat{\gamma}+\underline{\gamma})^{2}}{2(\hat{\kappa}-\underline{\kappa})-2 L(\kappa-\bar{\kappa})}<1$. So there exists $u_{0}>1$ such that $(p-1) \frac{(\gamma+\bar{\gamma})^{2}}{2(\kappa-\bar{\kappa})} u_{0}+(p-1) \frac{(\hat{\gamma}+\underline{\gamma})^{2}}{2(\hat{\kappa}-\underline{\kappa})-2 L(\kappa-\bar{\kappa})}=1$. Let $\rho_{1}(u)=(p-1) \frac{(\gamma+\bar{\gamma})^{2}}{2(\kappa-\bar{\kappa})} u, u \in\left[1, u_{0}\right)$, satisfying $2(\kappa-\bar{\kappa}) \geq \frac{p-1}{\rho_{1}(u)}(\gamma+\bar{\gamma})^{2}, 2(\hat{\kappa}-\underline{\kappa})-\frac{(\hat{\gamma}+\underline{\gamma})^{2}}{1-\rho_{1}(u)}(p-1)>2(\kappa-\bar{\kappa}) L$. Using Lemma 2.3 , we get that there exists a constant $\bar{a}>0$ satisfying $\inf _{t \geq 0} G(|\mathbf{x}(t)|)>\bar{a}$. So we choose $\rho_{2}$ which is sufficiently close to 1 and sufficiently small $\varepsilon_{1}, \varepsilon_{2}$ such that

$$
\begin{aligned}
& \left(2(\hat{\kappa}-\underline{\kappa})-\frac{(p-1)(\hat{\gamma}+\underline{\gamma})^{2}}{\left(1-\rho_{1}\right) \rho_{2}}-\frac{2(p-2)}{p} \varepsilon_{1}^{p}-\frac{(p-2)}{p} \frac{p-1}{1-\rho_{1}} \frac{1}{1-\rho_{2}} \varepsilon_{2}^{p}\right) \\
& -\frac{p-1}{\rho_{1}}(\gamma+\bar{\gamma})^{2}|\mathbf{x}(t)|^{2 n_{2}-2}+2(\kappa-\bar{\kappa})|\mathbf{x}(t)|^{n_{1}-1}>\bar{a} .
\end{aligned}
$$

Thus, we have

$$
\begin{aligned}
\mathscr{L} V \leq & -\frac{p}{2} \bar{a}|\mathbf{x}(t)|^{p}+p \bar{\kappa} \frac{n_{1}+1}{p+n_{1}-1} J_{1}+2 \underline{\kappa} J_{2}+\frac{p}{2} \frac{(p-1)(\bar{\gamma}+\gamma) \bar{\gamma}}{\rho_{1}} \frac{2 n_{2}}{p+2 n_{2}-2} J_{3} \\
& +\frac{(p-1)(\underline{\gamma}+\hat{\gamma}) \underline{\gamma}}{1-\rho_{1}} J_{4}+2 \varepsilon_{1}^{-\frac{p(p-2)}{2}} \xi_{1}^{\frac{p}{2}}(t)+\frac{p-1}{1-\rho_{1}} \frac{1}{1-\rho_{2}} \varepsilon_{2}^{-\frac{p(p-2)}{2}} \xi_{2}^{p}(t) .
\end{aligned}
$$

By virtue of the fact that $\int_{0}^{t}\left(\int_{-\tau}^{0}|\mathbf{x}(s+\theta)|^{w_{i}} \mathrm{~d} \eta_{i}(\theta)-|\mathbf{x}(s)|^{w_{i}}\right) \mathrm{d} s \leq \int_{-\tau}^{0}|\mathbf{x}(s)|^{w_{i}} \mathrm{~d} s$ for $w_{1}=p+$ $n_{1}-1, w_{3}=p+2 n_{2}-2, w_{2}=w_{4}=p$, respectively, we have

$$
\begin{aligned}
E|\mathbf{x}(t)|^{p} \leq & E|\mathbf{x}(0)|^{p}-\frac{p}{2} \bar{a} \int_{0}^{t} E|\mathbf{x}(s)|^{p} \mathrm{~d} s+p \bar{\kappa} \frac{n_{1}+1}{p+n_{1}-1} \int_{-\tau}^{0} E|\mathbf{x}(s)|^{p+n_{1}-1} \mathrm{~d} s \\
& +\frac{p}{2} \frac{(p-1)(\bar{\gamma}+\gamma) \bar{\gamma}}{\rho_{1}} \frac{2 n_{2}}{p+2 n_{2}-2} \int_{-\tau}^{0} E|\mathbf{x}(s)|^{p+2 n_{2}-2} \mathrm{~d} s \\
& +\left(2 \underline{\kappa}+\frac{(p-1)(\underline{\gamma}+\hat{\gamma}) \underline{\gamma}}{1-\rho_{1}}\right) \\
& \times \int_{-\tau}^{0} E|\mathbf{x}(s)|^{p} \mathrm{~d} s+\int_{0}^{t}\left[\frac{p-1}{1-\rho_{1}} \frac{1}{1-\rho_{2}} \varepsilon_{2}^{-\frac{p(p-2)}{2}} \xi_{2}^{p}(s)+2 \varepsilon_{1}^{-\frac{p(p-2)}{2}} \xi_{1}^{\frac{p}{2}}(s)\right] \mathrm{d} s .
\end{aligned}
$$

From (8) and Lemma 2.2, we get that $\int_{0}^{\infty} E|\mathbf{x}(s)|^{p} \mathrm{~d} s<\infty$. Combining with Lemma 3.3, we claim that $E|\mathbf{x}(t)|^{p}$ is uniformly continuous on $[0, \infty)$ for any $p \in\left[2, p_{0}\right)$. Due to Lemma 2.1, we have

$$
\lim _{t \rightarrow \infty} E|\mathbf{x}(t)|^{p}=0
$$

For any $p \in(0,2)$, using Hölder's inequality, we get

$$
\lim _{t \rightarrow \infty} E|\mathbf{x}(t)|^{p} \leq \lim _{t \rightarrow \infty}\left(E|\mathbf{x}(t)|^{2}\right)^{\frac{p}{2}}=0
$$


Remark 3 Clearly, the key of the proof is the positive lower-boundedness of the function $G(|\mathbf{x}(t)|)$, which depends on condition (4) and the definition of $p_{0}$.

\section{Exponential stability of SFDEs}

To study exponential stability, we slightly modify the polynomial growth condition (Assumption 2.2) as follows.

Assumption 4.1 There exist constants $\kappa, \bar{\kappa}, \hat{\kappa}, \underline{\kappa}, \kappa_{0}, \gamma, \bar{\gamma}, \hat{\gamma}, \underline{\gamma}, \gamma_{0} \geq 0, \alpha_{1}, \alpha_{2}>0$, probability measures $\eta_{i}$ on [- $\left.\tau, 0\right], i=1,2,3,4$, and positive numbers $n_{1}>1, n_{2}>1$ satisfying $n_{1}+1>2 n_{2}$ such that

$$
\begin{aligned}
\boldsymbol{\varphi}(0)^{T} \mathbf{f}(\boldsymbol{\varphi}, t) \leq & -\kappa|\boldsymbol{\varphi}(0)|^{n_{1}+1}+\bar{\kappa} \int_{-\tau}^{0}|\boldsymbol{\varphi}(\theta)|^{n_{1}+1} \mathrm{~d} \eta_{1}(\theta)-\hat{\kappa}|\boldsymbol{\varphi}(0)|^{2} \\
& +\underline{\kappa} \int_{-\tau}^{0}|\boldsymbol{\varphi}(\theta)|^{2} \mathrm{~d} \eta_{2}(\theta)+\kappa_{0} e^{-\alpha_{1} t}
\end{aligned}
$$

for all $\varphi \in C\left([-\tau, 0] ; R^{n}\right), t \in R_{+}$.

Remark 4 It is obvious that Assumption 4.1 is only a special case of Assumption 2.2.

In this section, our aim is to study the exponential stability of system (1). We have the following theorem.

Theorem 4.1 If Assumptions 2.1, 4.1 and condition (4) hold, then for any initial data $\zeta \in$ $C$, there is a unique global solution $\mathbf{x}(t, \zeta)$ of system (1) on $t \geq-\tau$, and $\mathbf{x}(t, \zeta)$ is almost surely exponentially stable and pth moment exponentially stable for any $p \in\left(0, p_{0}\right)$, namely

$$
\begin{aligned}
& \limsup _{t \rightarrow \infty} \frac{\log (|\mathbf{x}(t, \zeta)|)}{t} \leq-\frac{\varepsilon_{p}}{p}, \quad p \in\left[2, p_{0}\right) \text { a.s., } \\
& \limsup _{t \rightarrow \infty} \frac{\log \left(E|\mathbf{x}(t, \zeta)|^{p}\right)}{t} \leq-\varepsilon_{p}, \quad p \in\left[2, p_{0}\right), \\
& \limsup _{t \rightarrow \infty} \frac{\log \left(E|\mathbf{x}(t, \zeta)|^{p}\right)}{t} \leq-\frac{p}{2} \varepsilon_{2}, \quad p \in(0,2),
\end{aligned}
$$

where $p_{0}$ is the same as defined in Theorem 3.1, and $\varepsilon_{p}$ is a positive constant which only depends on $p$ but not on $\zeta$.

Proof From condition (4) and the definition of $p_{0}$, we obtain $(p-1) \frac{(\gamma+\bar{\gamma})^{2}}{2(\kappa-\bar{\kappa})}+(p-1) \times$ $\frac{(\hat{\gamma}+\underline{\gamma})^{2}}{2(\hat{\kappa}-L \kappa)-2(\underline{\kappa}-L \bar{\kappa})}<1$ for any $p \in\left[2, p_{0}\right)$. Then there exists at least a sufficiently small positive constant $\varepsilon$ satisfying $(p-1) \frac{(\underline{\gamma}+\bar{\gamma})\left(\gamma+\bar{\gamma} e^{\varepsilon \tau}\right)}{2\left(\kappa-\bar{\kappa} e^{\varepsilon \tau}\right)}+(p-1) \frac{(\hat{\gamma}+\underline{\gamma})\left(\hat{\gamma}+\underline{\underline{\gamma}} e^{\varepsilon \tau}\right)}{2(\hat{\kappa}-L \kappa)-\frac{2}{\bar{p}} \varepsilon-2(\underline{\kappa}-L \bar{\kappa}) e^{\varepsilon \tau}}<1,(\hat{\kappa}-L \kappa)-\frac{\varepsilon}{p}-(\underline{\kappa}-$ $L \bar{\kappa}) e^{\varepsilon \tau}>0, \kappa-\bar{\kappa} e^{\varepsilon \tau}>0, \varepsilon<\frac{p \alpha_{1}}{2}, \varepsilon<p \alpha_{2}$. So, by the continuity, define

$$
\begin{aligned}
\varepsilon_{p}= & \sup \left\{\varepsilon>0: \frac{(\gamma+\bar{\gamma})\left(\gamma+\bar{\gamma} e^{\varepsilon \tau}\right)}{2\left(\kappa-\bar{\kappa} e^{\varepsilon \tau}\right)}+\frac{(\hat{\gamma}+\underline{\gamma})\left(\hat{\gamma}+\underline{\gamma} e^{\varepsilon \tau}\right)}{2(\hat{\kappa}-L \kappa)-\frac{2}{p} \varepsilon-2(\underline{\kappa}-L \bar{\kappa}) e^{\varepsilon \tau}}<\frac{1}{p-1},\right. \\
& \left.(\hat{\kappa}-L \kappa)-\frac{\varepsilon}{p}-(\underline{\kappa}-L \bar{\kappa}) e^{\varepsilon \tau}>0, \kappa-\bar{\kappa} e^{\varepsilon \tau}>0, \varepsilon<\frac{p \alpha_{1}}{2}, \varepsilon<p \alpha_{2}\right\} .
\end{aligned}
$$


From Lemma 3.1 and condition (4), there exists a unique global solution. For the sake of simplicity, write $\mathbf{x}(t)=\mathbf{x}(t, \zeta)$. For any $p \in\left[2, p_{0}\right)$, applying Itô's formula to $V(\mathbf{x}, t)=$ $e^{\varepsilon t}|\mathbf{x}(t)|^{p}, \varepsilon \in\left(0, \varepsilon_{p}\right]$ and using the elemental inequalities, we have

$$
\begin{aligned}
& \mathscr{L}|\mathbf{x}(t)|^{p}+\varepsilon|\mathbf{x}(t)|^{p} \\
& \leq \varepsilon|\mathbf{x}(t)|^{p}+\frac{p}{2}|\mathbf{x}(t)|^{p-2}\left[-2 \kappa|\mathbf{x}(t)|^{n_{1}+1}-2 \hat{\kappa}|\mathbf{x}(t)|^{2}+2 \bar{\kappa} \int_{-\tau}^{0}|\mathbf{x}(t+\theta)|^{n_{1}+1} \mathrm{~d} \eta_{1}(\theta)\right. \\
& +2 \underline{\kappa} \int_{-\tau}^{0}|\mathbf{x}(t+\theta)|^{2} \mathrm{~d} \eta_{2}(\theta)+\frac{p-1}{\rho_{1}}\left(\frac{\gamma^{2}}{\delta_{1}}|\mathbf{x}(t)|^{2 n_{2}}+\frac{\bar{\gamma}^{2}}{1-\delta_{1}} \int_{-\tau}^{0}|\mathbf{x}(t+\theta)|^{2 n_{2}} \mathrm{~d} \eta_{3}(\theta)\right) \\
& +\frac{p-1}{\rho_{2}\left(1-\rho_{1}\right)}\left(\frac{\hat{\gamma}^{2}}{\delta_{2}}|\mathbf{x}(t)|^{2}+\frac{\underline{\gamma}^{2}}{1-\delta_{2}} \int_{-\tau}^{0}|\mathbf{x}(t+\theta)|^{2} \mathrm{~d} \eta_{4}(\theta)\right) \\
& \left.+2 \kappa_{0} e^{-\alpha_{1} t}+\frac{p-1}{\left(1-\rho_{1}\right)\left(1-\rho_{2}\right)} \gamma_{0}^{2} e^{-2 \alpha_{2} t}\right] \\
& \leq \frac{p}{2}\left[-2\left(\kappa-\bar{\kappa}\left(\frac{p-2}{p+n_{1}-1}+\frac{n_{1}+1}{p+n_{1}-1} e^{\varepsilon \tau}\right)\right)|\mathbf{x}(t)|^{p+n_{1}-1}\right. \\
& -\left(2 \hat{\kappa}-2 \underline{\kappa}\left(\frac{p-2}{p}+\frac{2}{p} e^{\varepsilon \tau}\right)-\frac{p-1}{\rho_{2}\left(1-\rho_{1}\right)}\left(\frac{\hat{\gamma}^{2}}{\delta_{2}}+\frac{\underline{\gamma}^{2}}{1-\delta_{2}}\left(\frac{p-2}{p}+\frac{2}{p} e^{\varepsilon \tau}\right)\right)\right. \\
& \left.-2 \frac{p-2}{p} \varepsilon_{1}^{p}-\frac{p-1}{1-\rho_{1}} \frac{1}{1-\rho_{2}} \frac{p-2}{p} \varepsilon_{2}^{p}-\frac{2}{p} \varepsilon\right)|\mathbf{x}(t)|^{p} \\
& \left.+\frac{p-1}{\rho_{1}}\left(\frac{\gamma^{2}}{\delta_{1}}+\frac{\bar{\gamma}^{2}}{1-\delta_{1}}\left(\frac{p-2}{p+2 n_{2}-2}+\frac{2 n_{2}}{p+2 n_{2}-2} e^{\varepsilon \tau}\right)\right)|\mathbf{x}(t)|^{p+2 n_{2}-2}\right] \\
& +2 \underline{\kappa} J_{2}+\frac{p-1}{\rho_{2}\left(1-\rho_{1}\right)} \frac{\underline{\gamma}^{2}}{1-\delta_{2}} J_{4}+\frac{p}{2} \frac{p-1}{\rho_{1}} \frac{\bar{\gamma}^{2}}{1-\delta_{1}} \frac{2 n_{2}}{p+2 n_{2}-2} J_{3}+p \bar{\kappa} \frac{n_{1}+1}{p+n_{1}-1} J_{1} \\
& +2 \varepsilon_{1}^{-\frac{p(p-2)}{2}}\left(\kappa_{0}^{\frac{p}{2}} e^{-\frac{p}{2} \alpha_{1} t}\right)+\frac{p-1}{1-\rho_{1}} \frac{1}{1-\rho_{2}} \varepsilon_{2}^{-\frac{p(p-2)}{2}}\left(\gamma_{0}^{p} e^{-p \alpha_{2} t}\right) \\
& \leq \frac{p}{2}\left[-2\left(\kappa-\bar{\kappa} e^{\varepsilon \tau}\right)|\mathbf{x}(t)|^{p+n_{1}-1}-\left(2 \hat{\kappa}-2 \underline{\kappa} e^{\varepsilon \tau}-\frac{p-1}{\rho_{2}\left(1-\rho_{1}\right)}\left(\frac{\hat{\gamma}^{2}}{\delta_{2}}+\frac{\underline{\gamma}^{2}}{1-\delta_{2}} e^{\varepsilon \tau}\right)\right.\right. \\
& \left.-2 \frac{p-2}{p} \varepsilon_{1}^{p}-\frac{p-1}{1-\rho_{1}} \frac{1}{1-\rho_{2}} \frac{p-2}{p} \varepsilon_{2}^{p}-\frac{2}{p} \varepsilon\right)|\mathbf{x}(t)|^{p}+\frac{p-1}{\rho_{1}}\left(\frac{\gamma^{2}}{\delta_{1}}+\frac{\bar{\gamma}^{2}}{1-\delta_{1}} e^{\varepsilon \tau}\right) \\
& \left.\times|\mathbf{x}(t)|^{p+2 n_{2}-2}\right]+2 \underline{\kappa} J_{2}+\frac{p-1}{\rho_{2}\left(1-\rho_{1}\right)} \frac{\underline{\gamma}^{2}}{1-\delta_{2}} J_{4}+\frac{p}{2} \frac{p-1}{\rho_{1}} \frac{\bar{\gamma}^{2}}{1-\delta_{1}} \frac{2 n_{2}}{p+2 n_{2}-2} J_{3} \\
& +p \bar{\kappa} \frac{n_{1}+1}{p+n_{1}-1} J_{1}+2 \varepsilon_{1}^{-\frac{p(p-2)}{2}}\left(\kappa_{0}^{\frac{p}{2}} e^{-\frac{p}{2} \alpha_{1} t}\right)+\frac{p-1}{1-\rho_{1}} \frac{1}{1-\rho_{2}} \varepsilon_{2}^{-\frac{p(p-2)}{2}}\left(\gamma_{0}^{p} e^{-p \alpha_{2} t}\right) \text {, }
\end{aligned}
$$

where $\rho_{1}, \rho_{2}, \delta_{1}, \delta_{2} \in(0,1), J_{1}=\int_{-\tau}^{0}|\mathbf{x}(t+\theta)|^{p+n_{1}-1} \mathrm{~d} \eta_{1}(\theta)-e^{\varepsilon \tau}|\mathbf{x}(t)|^{p+n_{1}-1}, J_{2}=\int_{-\tau}^{0} \mid \mathbf{x}(t+$ $\theta)\left.\right|^{p} \mathrm{~d} \eta_{2}(\theta)-e^{\varepsilon \tau}|\mathbf{x}(t)|^{p}, J_{3}=\int_{-\tau}^{0}|\mathbf{x}(t+\theta)|^{p+2 n_{2}-2} \mathrm{~d} \eta_{3}(\theta)-e^{\varepsilon \tau}|\mathbf{x}(t)|^{p+2 n_{2}-2}, J_{4}=\int_{-\tau}^{0} \mid \mathbf{x}(t+$ $\theta)\left.\right|^{p} \mathrm{~d} \eta_{4}(\theta)-e^{\varepsilon \tau}|\mathbf{x}(t)|^{p}$. Setting $\delta_{1}=\frac{\gamma}{\gamma+\bar{\gamma}}, \delta_{2}=\frac{\hat{\gamma}}{\hat{\gamma}+\underline{\gamma}}$, we arrive at

$$
\begin{aligned}
\mathscr{L}|\mathbf{x}(t)|^{p}+\varepsilon|\mathbf{x}(t)|^{p} \\
\leq \frac{p}{2}\left[-2\left(\kappa-\bar{\kappa} e^{\varepsilon \tau}\right)|\mathbf{x}(t)|^{p+n_{1}-1}-\left(2 \hat{\kappa}-2 \underline{\kappa} e^{\varepsilon \tau}-\frac{(p-1)(\hat{\gamma}+\underline{\gamma})\left(\hat{\gamma}+\underline{\gamma} e^{\varepsilon \tau}\right)}{\rho_{2}\left(1-\rho_{1}\right)}\right.\right. \\
\left.\quad-2 \frac{p-2}{p} \varepsilon_{1}^{p}-\frac{p-1}{1-\rho_{1}} \frac{1}{1-\rho_{2}} \frac{p-2}{p} \varepsilon_{2}^{p}-\frac{2}{p} \varepsilon\right)|\mathbf{x}(t)|^{p}
\end{aligned}
$$




$$
\begin{aligned}
& \left.+\frac{(p-1)(\gamma+\bar{\gamma})\left(\gamma+\bar{\gamma} e^{\varepsilon \tau}\right)}{\rho_{1}}|\mathbf{x}(t)|^{p+2 n_{2}-2}\right]+p \bar{\kappa} \frac{n_{1}+1}{p+n_{1}-1} J_{1}+(2 \underline{\kappa}) J_{2} \\
& +\frac{p}{2} \frac{(p-1)(\bar{\gamma}+\gamma) \bar{\gamma}}{\rho_{1}} \frac{2 n_{2}}{p+2 n_{2}-2} J_{3}+\frac{(p-1)(\underline{\gamma}+\hat{\gamma}) \underline{\gamma}}{\rho_{2}\left(1-\rho_{1}\right)} J_{4}+2 \varepsilon_{1}^{-\frac{p(p-2)}{2}} \kappa_{0}^{\frac{p}{2}} e^{-\frac{p}{2} \alpha_{1} t} \\
& +\frac{p-1}{1-\rho_{1}} \frac{1}{1-\rho_{2}} \varepsilon_{2}^{-\frac{p(p-2)}{2}} \gamma_{0}^{p} e^{-p \alpha_{2} t} .
\end{aligned}
$$

Let $H(|\mathbf{x}(t)|)=\left(2 \hat{\kappa}-2 \underline{\kappa} e^{\varepsilon \tau}-\frac{(p-1)\left((\hat{\gamma}+\underline{\gamma})\left(\hat{\gamma}+\underline{\gamma} e^{\varepsilon \tau}\right)\right)}{\left(1-\rho_{1}\right)}-\frac{2}{p} \varepsilon\right)+2\left(\kappa-\bar{\kappa} e^{\varepsilon \tau}\right)|\mathbf{x}(t)|^{n_{1}-1}-\frac{(p-1)(\gamma+\bar{\gamma})\left(\gamma+\bar{\gamma} e^{\varepsilon \tau}\right)}{\rho_{1}} \times$ $|\mathbf{x}(t)|^{2 n_{2}-2}$. By the same technique as the function $G(|\mathbf{x}(t)|)$ in Theorem 3.1, we get that there exists $\overline{\bar{a}}>0$ satisfying $\inf _{t \geq 0} H(|\mathbf{x}(t)|)>\overline{\bar{a}}$. So we choose $\rho_{2}$ which is sufficiently close to 1 and sufficiently small $\varepsilon_{1}, \varepsilon_{2}$ such that

$$
\begin{aligned}
& \left(2 \hat{\kappa}-2 \underline{\kappa} e^{\varepsilon \tau}-\frac{(p-1)\left((\hat{\gamma}+\underline{\gamma})\left(\hat{\gamma}+\underline{\gamma} e^{\varepsilon \tau}\right)\right)}{\rho_{2}\left(1-\rho_{1}\right)}-2 \frac{p-2}{p} \varepsilon_{1}^{p}-\frac{p-1}{1-\rho_{1}} \frac{1}{1-\rho_{2}} \frac{p-2}{p} \varepsilon_{2}^{p}-\frac{2}{p} \varepsilon\right) \\
& +2\left(\kappa-\bar{\kappa} e^{\varepsilon \tau}\right)|\mathbf{x}(t)|^{n_{1}-1}-\frac{(p-1)(\gamma+\bar{\gamma})\left(\gamma+\bar{\gamma} e^{\varepsilon \tau}\right)}{\rho_{1}}|\mathbf{x}(t)|^{2 n_{2}-2}>\overline{\bar{a}} .
\end{aligned}
$$

Hence, we have

$$
\begin{aligned}
\mathscr{L}|\mathbf{x}(t)|^{p}+\varepsilon|\mathbf{x}(t)|^{p} & \\
\leq & -\frac{p}{2} \overline{\bar{a}}|\mathbf{x}(t)|^{p}+p \bar{\kappa} \frac{n_{1}+1}{p+n_{1}-1} J_{1}+2 \underline{\kappa} J_{2}+\frac{p}{2} \frac{(p-1)(\bar{\gamma}+\gamma) \bar{\gamma}}{\rho_{1}} \frac{2 n_{2}}{p+2 n_{2}-2} J_{3} \\
& +\frac{(p-1)(\underline{\gamma}+\hat{\gamma}) \underline{\gamma}}{1-\rho_{1}} J_{4}+2 \varepsilon_{1}^{-\frac{p(p-2)}{2}} \kappa_{0}^{\frac{p}{2}} e^{-\frac{p}{2} \alpha_{1} t}+\frac{p-1}{1-\rho_{1}} \frac{1}{1-\rho_{2}} \varepsilon_{2}^{-\frac{p(p-2)}{2}} \gamma_{0}^{p} e^{-p \alpha_{2} t} .
\end{aligned}
$$

By virtue of the fact that

$$
\int_{0}^{t} e^{\varepsilon s} J_{i} \mathrm{~d} s=\int_{0}^{t} e^{\varepsilon s}\left(\int_{-\tau}^{0}|\mathbf{x}(s+\theta)|^{w_{i}} \mathrm{~d} \eta_{i}(\theta)-e^{\varepsilon \tau}|\mathbf{x}(s)|^{w_{i}}\right) \mathrm{d} s \leq e^{\varepsilon \tau} \int_{-\tau}^{0}|\mathbf{x}(s)|^{w_{i}} \mathrm{~d} s
$$

for $w_{1}=p+n_{1}-1, w_{3}=p+2 n_{2}-2, w_{2}=w_{4}=p$, respectively, and using Itô's formula to $V(\mathbf{x}, t)=e^{\varepsilon t}|\mathbf{x}(t)|^{p}, \varepsilon \in\left(0, \varepsilon_{p}\right]$, we have

$$
\begin{aligned}
e^{\varepsilon t}|\mathbf{x}(t)|^{p}= & |\mathbf{x}(0)|^{p}+\int_{0}^{t} e^{\varepsilon s}\left(\mathscr{L}|\mathbf{x}(s)|^{p}+\varepsilon|\mathbf{x}(s)|^{p}\right) \mathrm{d} s+M(t) \\
\leq & |\mathbf{x}(0)|^{p}-\frac{p}{2} \overline{\bar{a}} \int_{0}^{t} e^{\varepsilon s}|\mathbf{x}(s)|^{p} \mathrm{~d} s+\frac{p}{2} \frac{(p-1)(\bar{\gamma}+\gamma) \bar{\gamma}}{\rho_{1}} \frac{2 n_{2}}{p+2 n_{2}-2} \int_{0}^{t} e^{\varepsilon s} J_{3} \mathrm{~d} s \\
& +p \bar{\kappa} \frac{n_{1}+1}{p+n_{1}-1} \int_{0}^{t} e^{\varepsilon s} J_{1} \mathrm{~d} s+2 \underline{\kappa} \int_{0}^{t} e^{\varepsilon s} J_{2} \mathrm{~d} s+\frac{(p-1)(\underline{\gamma}+\hat{\gamma}) \underline{\gamma}}{1-\rho_{1}} \int_{0}^{t} e^{\varepsilon s} J_{4} \mathrm{~d} s \\
& +2 \varepsilon_{1}^{-\frac{p(p-2)}{2}} \kappa_{0}^{\frac{p}{2}} \int_{0}^{t} e^{\left(\varepsilon-\frac{p}{2} \alpha_{1}\right) s} \mathrm{~d} s \\
& +\frac{p-1}{1-\rho_{1}} \frac{1}{1-\rho_{2}} \varepsilon_{2}^{-\frac{p(p-2)}{2}} \gamma_{0}^{p} \int_{0}^{t} e^{\left(\varepsilon-p \alpha_{2}\right) s} \mathrm{~d} s+M(t) \\
\leq & |\mathbf{x}(0)|^{p}-\frac{p}{2} \overline{\bar{a}} \int_{0}^{t} e^{\varepsilon s}|\mathbf{x}(s)|^{p} \mathrm{~d} s \\
& +\frac{p}{2} \frac{(p-1)(\bar{\gamma}+\gamma) \bar{\gamma}}{\rho_{1}} \frac{2 n_{2}}{p+2 n_{2}-2} e^{\varepsilon \tau} \int_{-\tau}^{0}|\mathbf{x}(s)|^{p+2 n_{2}-2} \mathrm{~d} s
\end{aligned}
$$




$$
\begin{aligned}
& +p \bar{\kappa} \frac{n_{1}+1}{p+n_{1}-1} e^{\varepsilon \tau} \int_{-\tau}^{0}|\mathbf{x}(s)|^{p+n_{1}-1} \mathrm{~d} s \\
& +\left(2 \underline{\kappa}+\frac{(p-1)(\underline{\gamma}+\hat{\gamma}) \underline{\gamma}}{1-\rho_{1}}\right) e^{\varepsilon \tau} \int_{-\tau}^{0}|\mathbf{x}(s)|^{p} \mathrm{~d} s \\
& +2 \varepsilon_{1}^{-\frac{p(p-2)}{2}} \kappa_{0}^{\frac{p}{2}} \frac{1}{\left(\frac{p}{2} \alpha_{1}-\varepsilon\right)}+\frac{p-1}{1-\rho_{1}} \frac{1}{1-\rho_{2}} \varepsilon_{2}^{-\frac{p(p-2)}{2}} \gamma_{0}^{p} \frac{1}{\left(p \alpha_{2}-\varepsilon\right)}+M(t),
\end{aligned}
$$

where $M(t)=\int_{0}^{t} p e^{\varepsilon s}|\mathbf{x}(s)|^{p-1} g\left(\mathbf{x}_{s}, s\right) \mathrm{d} B(s)$ is a local martingale with the initial value $M(0)=0$. Applying the nonnegative semi-martingale convergence theorem (see Lemma 2.5), we obtain that

$$
\limsup _{t \rightarrow \infty} e^{\varepsilon t}|\mathbf{x}(t)|^{p}<\infty \quad \text { a.s. }
$$

Hence, there exists a finite positive random variable $\bar{\zeta}$ such that

$$
\sup _{0 \leq t<\infty} e^{\varepsilon t}|\mathbf{x}(t)|^{p}<\bar{\zeta} \quad \text { a.s. }
$$

So, letting $\varepsilon \rightarrow \varepsilon_{p}$ in the inequality above, we claim that

$$
\limsup _{t \rightarrow \infty} \frac{\log (|\mathbf{x}(t)|)}{t} \leq-\frac{\varepsilon_{p}}{p}, \quad p \in\left[2, p_{0}\right) \text { a.s. }
$$

which is the required result (11).

Next, we prove the result (12). From (15), we get

$$
\begin{aligned}
E e^{\varepsilon t}|\mathbf{x}(t)|^{p}= & E|\mathbf{x}(0)|^{p}+E \int_{0}^{t} e^{\varepsilon s}\left(\mathscr{L}|\mathbf{x}(s)|^{p}+\varepsilon|\mathbf{x}(s)|^{p}\right) \mathrm{d} s \\
\leq & E|\mathbf{x}(0)|^{p}-\frac{p}{2} \overline{\bar{a}} E \int_{0}^{t} e^{\varepsilon s}|\mathbf{x}(s)|^{p} \mathrm{~d} s+\frac{p}{2} \frac{(p-1)(\bar{\gamma}+\gamma) \bar{\gamma}}{\rho_{1}} \frac{2 n_{2}}{p+2 n_{2}-2} e^{\varepsilon \tau} \\
& \times \int_{-\tau}^{0} E|\mathbf{x}(s)|^{p+2 n_{2}-2} \mathrm{~d} s+p \bar{\kappa} \frac{n_{1}+1}{p+n_{1}-1} e^{\varepsilon \tau} \int_{-\tau}^{0} E|\mathbf{x}(s)|^{p+n_{1}-1} \mathrm{~d} s \\
& +\left(2 \underline{\kappa}+\frac{(p-1)(\underline{\gamma}+\hat{\gamma}) \underline{\gamma}}{1-\rho_{1}}\right) e^{\varepsilon \tau} \int_{-\tau}^{0} E|\mathbf{x}(s)|^{p} \mathrm{~d} s+2 \varepsilon_{1}^{-\frac{p(p-2)}{2}} \kappa_{0}^{\frac{p}{2}} \frac{1}{\left(\frac{p}{2} \alpha_{1}-\varepsilon\right)} \\
& +\frac{p-1}{1-\rho_{1}} \frac{1}{1-\rho_{2}} \varepsilon_{2}^{-\frac{p(p-2)}{2}} \gamma_{0}^{p} \frac{1}{\left(p \alpha_{2}-\varepsilon\right)} \\
\leq & c_{1},
\end{aligned}
$$

where $c_{1}=\frac{p}{2} \frac{(p-1)(\bar{\gamma}+\gamma) \bar{\gamma}}{\rho_{1}} \frac{2 n_{2}}{p+2 n_{2}-2} e^{\varepsilon \tau} \int_{-\tau}^{0} E|\mathbf{x}(s)|^{p+2 n_{2}-2} \mathrm{~d} s+p \bar{\kappa} \frac{n_{1}+1}{p+n_{1}-1} e^{\varepsilon \tau} \int_{-\tau}^{0} E|\mathbf{x}(s)|^{p+n_{1}-1} \mathrm{~d} s+$ $\left(2 \underline{\kappa}+\frac{(p-1)(\underline{\underline{\gamma}} \hat{\hat{\gamma}}) \underline{\underline{\gamma}}}{1-\rho_{1}}\right) e^{\varepsilon \tau} \int_{-\tau}^{0} E|\mathbf{x}(s)|^{p} \mathrm{~d} s+2 \varepsilon_{1}^{-\frac{p(p-2)}{2}} \kappa_{0}^{\frac{p}{2}} \frac{1}{\left(\frac{p}{2} \alpha_{1}-\varepsilon\right)}+\frac{p-1}{1-\rho_{1}} \frac{1}{1-\rho_{2}} \varepsilon_{2}^{-\frac{p(p-2)}{2}} \gamma_{0}^{p} \frac{1}{\left(p \alpha_{2}-\varepsilon\right)}+E|\mathbf{x}(0)|^{p}$. This implies

$$
E|\mathbf{x}(t)|^{p} \leq c_{1} e^{-\varepsilon t}, \quad \forall t>0 .
$$

So, letting $\varepsilon \rightarrow \varepsilon_{p}$ in the inequality above, we claim that, for $p \in\left[2, p_{0}\right)$,

$$
\limsup _{t \rightarrow \infty} \frac{\log \left(E|\mathbf{x}(t)|^{p}\right)}{t} \leq-\varepsilon_{p}
$$


For any $p \in(0,2)$, using Hölder's inequality, we obtain

$$
E|\mathbf{x}(t)|^{p} \leq\left(E|\mathbf{x}(t)|^{2}\right)^{\frac{p}{2}} \leq c_{2}^{\frac{p}{2}} e^{-\frac{p}{2} \varepsilon_{2} t}
$$

where $c_{2}=E|\mathbf{x}(0)|^{2}+\frac{(\bar{\gamma}+\gamma) \bar{\gamma}}{\rho_{1}} e^{\varepsilon \tau} \int_{-\tau}^{0} E|\mathbf{x}(s)|^{2 n_{2}} \mathrm{~d} s+\left(2 \underline{\kappa}+\frac{(\underline{\gamma}+\hat{\gamma}) \underline{\underline{\gamma}}}{1-\rho_{1}}\right) e^{\varepsilon \tau} \int_{-\tau}^{0} E|\mathbf{x}(s)|^{2} \mathrm{~d} s+2 \kappa_{0} \frac{1}{\left(\alpha_{1}-\varepsilon\right)}+$ $2 \bar{\kappa} e^{\varepsilon \tau} \int_{-\tau}^{0} E|\mathbf{x}(s)|^{n_{1}+1} \mathrm{~d} s+\frac{1}{1-\rho_{1}} \frac{1}{1-\rho_{2}} \gamma_{0}^{2} \frac{1}{\left(2 \alpha_{2}-\varepsilon\right)}$, which yields the assertion, for $p \in(0,2)$,

$$
\limsup _{t \rightarrow \infty} \frac{\log \left(E|\mathbf{x}(t)|^{p}\right)}{t} \leq-\frac{p}{2} \varepsilon_{2}
$$

which is the required result (12).

Remark 5 Since Assumption 4.1 implies Assumption 2.2, the conditions of Theorem 4.1 can guarantee the $p$ th moment asymptotic stability.

As a special case of Assumption 4.1, we obtain the following corollary directly from Theorem 4.1.

Corollary 4.1 If Assumptions 2.1, 2.2 and condition (4) hold, and the bounded functions $\xi_{1}(t), \xi_{2}(t)$ in Assumption 2.2 are 0 , then for any $p \in\left(0, p_{0}\right)$ and any initial data $\zeta \in C$, there is a unique global solution $\mathbf{x}(t, \zeta)$ of system (1) on $t \geq-\tau$, and $\mathbf{x}(t, \zeta)$ is almost surely exponentially stable and pth moment exponentially stable, namely

$$
\begin{aligned}
& \limsup _{t \rightarrow \infty} \frac{\log (|\mathbf{x}(t, \zeta)|)}{t} \leq-\frac{\varepsilon_{p}^{\prime}}{p}, \quad p \in\left[2, p_{0}\right) \text { a.s., } \\
& \limsup _{t \rightarrow \infty} \frac{\log \left(E|\mathbf{x}(t, \zeta)|^{p}\right)}{t} \leq-\varepsilon_{p}^{\prime}, \quad p \in\left[2, p_{0}\right), \\
& \limsup _{t \rightarrow \infty} \frac{\log \left(E|\mathbf{x}(t, \zeta)|^{p}\right)}{t} \leq-\frac{p}{2} \varepsilon_{2}^{\prime}, \quad p \in(0,2),
\end{aligned}
$$

where $p_{0}$ is the same as defined in Theorem 3.1, and $\varepsilon_{p}^{\prime}$ is a positive constant which only depends on $p$ but not on $\zeta$.

\section{Generalized theory on asymptotic stability of SFDEs}

Under Assumptions 2.2 and 4.1, we can see from the proofs of Theorems 3.1 and 4.1 that the positive lower-boundedness of the functions $G(|\mathbf{x}(t)|)$ and $H(|\mathbf{x}(t)|)$ play important roles, while condition (4) and the definition of $p_{0}$ are mainly used to ensure the positive lower-boundedness of the functions $G(|\mathbf{x}(t)|)$ and $H(|\mathbf{x}(t)|)$. So we wonder whether there exist more general assumptions and new conditions to guarantee these results. This is equivalent to finding more general assumptions and new conditions such that system (1) can still have the previous results, namely there exists a unique global solution almost surely, and the solution is asymptotically stable and, moreover, exponentially stable.

To make our theory more applicable, we replace the polynomial growth condition (Assumption 2.2) by the following general assumption.

Assumption 5.1 There are two functions $V \in C^{2,1}\left(R^{n} \times[-\tau, \infty) ; R_{+}\right), W \in C\left(R^{n} \times[-\tau\right.$, $\left.\infty) ; R_{+}\right)$and three probability measures $\eta_{i}$ on $[-\tau, 0]$ with $\int_{-\tau}^{0} \mathrm{~d} \eta_{i}(\theta)=1(i=1,2,3)$, as well 
as some constants $a_{0}, a_{1}, a_{2}, a_{3}, k_{1}, k_{2}, k_{3} \geq 0$, and $\beta_{2}>\beta_{1}>1$, such that

$$
\liminf _{|x| \rightarrow \infty, 0 \leq t<\infty} V(x, t)=\infty
$$

while for all $(\varphi, t) \in C\left([-\tau, 0] ; R^{n}\right) \times R_{+}$,

$$
\begin{aligned}
\mathscr{L} V(\varphi, t) \leq & a_{0}-a_{1} W^{\beta_{2}}(\varphi(0), t) \\
& +k_{1} \int_{-\tau}^{0} W^{\beta_{2}}(\varphi(\theta), t+\theta) \mathrm{d} \eta_{1}(\theta)+a_{2} W^{\beta_{1}}(\varphi(0), t) \\
& +k_{2} \int_{-\tau}^{0} W^{\beta_{1}}(\varphi(\theta), t+\theta) \mathrm{d} \eta_{2}(\theta)-a_{3} W(\varphi(0), t) \\
& +k_{3} \int_{-\tau}^{0} W(\varphi(\theta), t+\theta) \mathrm{d} \eta_{3}(\theta) .
\end{aligned}
$$

Remark 6 Condition (21) is known as radial unboundedness in the literature [20].

Remark 7 Compared with [22], our condition (22), different from their condition (5.4), emphasizes that $\mathscr{L} V$ is dominated by a polynomial function of $W$. What is more, the probability measures $\eta_{i}$ can also be weakened to any right-continuous nondecreasing functions (see [17]).

In the same way as [22], we can prove the following lemma.

Lemma 5.1 If Assumptions 2.1, 5.1 and $a_{1}>k_{1}$ hold, then for any initial data $\zeta \in C$, there is a unique global solution $\mathbf{x}(t, \zeta)$ of system (1) on $t \in[-\tau, \infty)$.

Now we examine the asymptotic stability and the exponential stability of system (1).

Theorem 5.1 If Assumptions 2.1, 5.1 and the following condition (23) hold,

$$
a_{1}-k_{1} \geq a_{2}+k_{2}>0, \quad a_{3}-k_{3}>\left(a_{1}-k_{1}\right) L^{\prime}, \quad a_{0}=0,
$$

except that (22) is replaced by

$$
\begin{aligned}
\mathscr{L} V(\varphi, t) \leq & a_{0}-a_{1} V^{\beta_{2}}(\varphi(0), t) \\
& +k_{1} \int_{-\tau}^{0} V^{\beta_{2}}(\varphi(\theta), t+\theta) \mathrm{d} \eta_{1}(\theta)+a_{2} V^{\beta_{1}}(\varphi(0), t) \\
& +k_{2} \int_{-\tau}^{0} V^{\beta_{1}}(\varphi(\theta), t+\theta) \mathrm{d} \eta_{2}(\theta)-a_{3} V(\varphi(0), t) \\
& +k_{3} \int_{-\tau}^{0} V(\varphi(\theta), t+\theta) \mathrm{d} \eta_{3}(\theta),
\end{aligned}
$$

then for any initial data $\zeta \in C$, there is a unique global solution $\mathbf{x}(t, \zeta)$ of system (1) on $t \in[-\tau, \infty)$, and $V(\mathbf{x}(t, \zeta), t)$ has the following properties:

(i) If $V(\mathbf{x}(t, \zeta), t)$ is uniformly continuous about $t$ on $[0, \infty)$, then

$$
\lim _{t \rightarrow \infty} V(\mathbf{x}(t, \zeta), t)=0 \quad \text { a.s. }
$$


(ii) If $E V(\mathbf{x}(t, \zeta), t)$ is uniformly continuous about $t$ on $[0, \infty)$, then

$$
\lim _{t \rightarrow \infty} E V(\mathbf{x}(t, \zeta), t)=0
$$

where $L^{\prime}=\left(\beta_{2}-\beta_{1}\right)\left(\beta_{2}-1\right)^{-\frac{\beta_{2}-1}{\beta_{2}-\beta_{1}}}\left(\beta_{1}-1\right)^{\frac{\beta_{1}-1}{\beta_{2}-\beta_{1}}}$

Proof The proof is similar to Theorem 3.1. From Lemma 5.1 and condition (23), there exists a unique global solution. For the sake of simplicity, write $\mathbf{x}(t)=\mathbf{x}(t, \zeta)$. Applying Itô's formula to $V(\mathbf{x}(t), t)$, we have

$$
\begin{aligned}
\mathscr{L} V\left(\mathbf{x}_{t}, t\right) \leq & -\left(a_{1}-k_{1}\right) V^{\beta_{2}}(\mathbf{x}(t), t)+\left(a_{2}+k_{2}\right) V^{\beta_{1}}(\mathbf{x}(t), t)-\left(a_{3}-k_{3}\right) V(\mathbf{x}(t), t) \\
& +k_{1} J_{1}+k_{2} J_{2}+k_{3} J_{3},
\end{aligned}
$$

where $J_{1}=\int_{-\tau}^{0} V^{\beta_{2}}(\mathbf{x}(t+\theta), t+\theta) \mathrm{d} \eta_{1}(\theta)-V^{\beta_{2}}(\mathbf{x}(t), t), J_{2}=\int_{-\tau}^{0} V^{\beta_{1}}(\mathbf{x}(t+\theta), t+\theta) \mathrm{d} \eta_{2}(\theta)-$ $V^{\beta_{1}}(\mathbf{x}(t), t), J_{3}=\int_{-\tau}^{0} V(\mathbf{x}(t+\theta), t+\theta) \mathrm{d} \eta_{3}(\theta)-V(\mathbf{x}(t), t)$.

Let $Q(V(\mathbf{x}(t), t))=\left(a_{1}-k_{1}\right) V^{\beta_{2}-1}(\mathbf{x}(t), t)-\left(a_{2}+k_{2}\right) V^{\beta_{1}-1}(\mathbf{x}(t), t)+\left(a_{3}-k_{3}\right)$. By the same technique as function $G(|\mathbf{x}(t)|)$ in Theorem 3.1, there exists a constant $\bar{Q}>0$ satisfying $\inf _{t \geq 0} Q(V(\mathbf{x}(t), t))>\bar{Q}$. Hence, we have

$$
\mathscr{L} V\left(\mathbf{x}_{t}, t\right) \leq-\bar{Q} V(\mathbf{x}(t), t)+k_{1} J_{1}+k_{2} J_{2}+k_{3} J_{3} .
$$

By virtue of the fact that

$$
\begin{aligned}
\int_{0}^{t} J_{i} \mathrm{~d} s & =\int_{0}^{t}\left(\int_{-\tau}^{0} V^{w_{i}^{\prime}}(\mathbf{x}(s+\theta), s+\theta) \mathrm{d} \eta_{i}(\theta)-V^{w_{i}^{\prime}}(\mathbf{x}(s), s)\right) \mathrm{d} s \\
& \leq \int_{-\tau}^{0} V^{w_{i}^{\prime}}(\mathbf{x}(s), s) \mathrm{d} s
\end{aligned}
$$

for $w_{1}^{\prime}=\beta_{2}, w_{2}^{\prime}=\beta_{1}, w_{3}^{\prime}=1$, respectively, and using Itô's formula, we have

$$
\begin{aligned}
V(\mathbf{x}(t), t)= & V(\mathbf{x}(0), 0)+\int_{0}^{t} \mathscr{L} V\left(\mathbf{x}_{s}, s\right) \mathrm{d} s+M_{1}(t) \\
\leq & V(\mathbf{x}(0), 0)-\bar{Q} \int_{0}^{t} V(\mathbf{x}(s), s) \mathrm{d} s+k_{1} \int_{-\tau}^{0} V^{\beta_{2}}(\mathbf{x}(s), s) \mathrm{d} s \\
& +k_{2} \int_{-\tau}^{0} V^{\beta_{1}}(\mathbf{x}(s), s) \mathrm{d} s+k_{3} \int_{-\tau}^{0} V(\mathbf{x}(s), s) \mathrm{d} s+M_{1}(t),
\end{aligned}
$$

where $M_{1}(t)=\int_{0}^{t} V_{x}(\mathbf{x}(s), s) g\left(\mathbf{x}_{s}, s\right) \mathrm{d} B(s)$ is a local martingale with the initial value $M_{1}(0)=0$. Applying the nonnegative semi-martingale convergence theorem (see Lemma 2.5), we obtain that

$$
\limsup _{t \rightarrow \infty} V(\mathbf{x}(t), t)<\infty \quad \text { a.s., } \quad \int_{0}^{\infty} V(\mathbf{x}(s), s) \mathrm{d} s<\infty .
$$

Hence, from Lemma 2.1 and the uniform continuity of $V(\mathbf{x}(t), t)$, we get the required result (25)

$$
\lim _{t \rightarrow \infty} V(\mathbf{x}(t), t)=0 \quad \text { a.s. }
$$


Next, we prove the result (26). From (28), we get that

$$
\begin{aligned}
E V(\mathbf{x}(t), t)= & E V(\mathbf{x}(0), 0)+E \int_{0}^{t} \mathscr{L} V\left(\mathbf{x}_{s}, s\right) \mathrm{d} s \\
\leq & E V(\mathbf{x}(0), 0)-\bar{Q} E \int_{0}^{t} V(\mathbf{x}(s), s) \mathrm{d} s+k_{1} E \int_{-\tau}^{0} V^{\beta_{2}}(\mathbf{x}(s), s) \mathrm{d} s \\
& +k_{2} E \int_{-\tau}^{0} V^{\beta_{1}}(\mathbf{x}(s), s) \mathrm{d} s+k_{3} E \int_{-\tau}^{0} V(\mathbf{x}(s), s) \mathrm{d} s .
\end{aligned}
$$

This implies

$$
E \int_{0}^{\infty} V(\mathbf{x}(s), s) \mathrm{d} s=\int_{0}^{\infty} E V(\mathbf{x}(s), s) \mathrm{d} s<\infty
$$

Hence, from Lemma 2.1 and the uniform continuity of $E V(\mathbf{x}(t), t)$, we have

$$
\lim _{t \rightarrow \infty} E V(\mathbf{x}(t), t)=0
$$

which is the required result (26).

Theorem 5.2 If Assumptions 2.1, 5.1 and condition (23) hold, except that (22) is replaced by (24), then for any initial data $\zeta \in C$, there is a unique global solution $\mathbf{x}(t, \zeta)$ of system (1) on $t \in[-\tau, \infty)$, and $V(\mathbf{x}(t, \zeta), t)$ has the following properties:

$$
\begin{aligned}
& \limsup _{t \rightarrow \infty} \frac{\log (V(\mathbf{x}(t, \zeta), t))}{t} \leq-\bar{\varepsilon} \quad \text { a.s. } \\
& \limsup _{t \rightarrow \infty} \frac{\log (E V(\mathbf{x}(t, \zeta), t))}{t} \leq-\bar{\varepsilon}
\end{aligned}
$$

where $\bar{\varepsilon}$ is a positive constant which does not depend on $\zeta$.

Proof The proof is similar to Theorem 4.1. From condition (23), we obtain that there exists at least a sufficiently small positive constant $\varepsilon$ satisfying $a_{1}-k_{1} e^{\varepsilon \tau} \geq a_{2}+k_{2} e^{\varepsilon \tau}>0$, $\left(a_{3}-\right.$ $\left.a_{1} L^{\prime}\right)-\varepsilon-\left(k_{3}-k_{1} L^{\prime}\right) e^{\varepsilon \tau}>0$. So, by the continuity, define

$$
\bar{\varepsilon}=\sup \left\{\varepsilon>0: a_{1}-k_{1} e^{\varepsilon \tau} \geq a_{2}+k_{2} e^{\varepsilon \tau}>0,\left(a_{3}-a_{1} L^{\prime}\right)-\varepsilon-\left(k_{3}-k_{1} L^{\prime}\right) e^{\varepsilon \tau}>0\right\} .
$$

From Lemma 5.1 and condition (23), there exists a unique global solution. For the sake of simplicity, write $\mathbf{x}(t)=\mathbf{x}(t, \zeta)$. Applying Itô's formula to $e^{\varepsilon t} V(\mathbf{x}(t), t), \varepsilon \in(0, \bar{\varepsilon}]$, we have

$$
\begin{aligned}
\mathscr{L} V\left(\mathbf{x}_{t}, t\right)+\varepsilon V(\mathbf{x}(t), t) \\
\leq-a_{1} V^{\beta_{2}}(\mathbf{x}(t), t)+k_{1} \int_{-\tau}^{0} V^{\beta_{2}}(\mathbf{x}(t+\theta), t+\theta) \mathrm{d} \eta_{1}(\theta) \\
\quad+a_{2} V^{\beta_{1}}(\mathbf{x}(t), t)+k_{2} \int_{-\tau}^{0} V^{\beta_{1}}(\mathbf{x}(t+\theta), t+\theta) \mathrm{d} \eta_{2}(\theta) \\
\quad-\left(a_{3}-\varepsilon\right) V(\mathbf{x}(t), t)+k_{3} \int_{-\tau}^{0} V(\mathbf{x}(t+\theta), t+\theta) \mathrm{d} \eta_{3}(\theta)
\end{aligned}
$$




$$
\begin{aligned}
\leq & -\left(a_{1}-k_{1} e^{\varepsilon \tau}\right) V^{\beta_{2}}(\mathbf{x}(t), t)+\left(a_{2}+k_{2} e^{\varepsilon \tau}\right) V^{\beta_{1}}(\mathbf{x}(t), t) \\
& -\left(a_{3}-k_{3} e^{\varepsilon \tau}-\varepsilon\right) V(\mathbf{x}(t), t)+k_{1} J_{1}+k_{2} J_{2}+k_{3} J_{3},
\end{aligned}
$$

where $J_{1}=\int_{-\tau}^{0} V^{\beta_{2}}(\mathbf{x}(t+\theta), t+\theta) \mathrm{d} \eta_{1}(\theta)-e^{\varepsilon \tau} V^{\beta_{2}}(\mathbf{x}(t), t), J_{2}=\int_{-\tau}^{0} V^{\beta_{1}}(\mathbf{x}(t+\theta), t+\theta) \mathrm{d} \eta_{2}(\theta)-$ $e^{\varepsilon \tau} V^{\beta_{1}}(\mathbf{x}(t), t), J_{3}=\int_{-\tau}^{0} V(\mathbf{x}(t+\theta), t+\theta) \mathrm{d} \eta_{3}(\theta)-e^{\varepsilon \tau} V(\mathbf{x}(t), t)$.

Let $R(V(\mathbf{x}(t), t))=\left(a_{1}-k_{1} e^{\varepsilon \tau}\right) V^{\beta_{2}-1}(\mathbf{x}(t), t)-\left(a_{2}+k_{2} e^{\varepsilon \tau}\right) V^{\beta_{1}-1}(\mathbf{x}(t), t)+\left(a_{3}-k_{3} e^{\varepsilon \tau}-\varepsilon\right)$. Noting the definition of $\bar{\varepsilon}$ and by the same technique as the function $G(|\mathbf{x}(t)|)$ in Theorem 3.1, there exists a constant $\bar{R}>0$ satisfying $\inf _{t \geq 0} R(V(\mathbf{x}(t), t))>\bar{R}$. Therefore, we have

$$
\mathscr{L} V\left(\mathbf{x}_{t}, t\right)+\varepsilon V(\mathbf{x}(t), t) \leq-\bar{R} V(\mathbf{x}(t), t)+k_{1} J_{1}+k_{2} J_{2}+k_{3} J_{3} .
$$

By virtue of the fact that

$$
\begin{aligned}
\int_{0}^{t} e^{\varepsilon s} J_{i} \mathrm{~d} s & =\int_{0}^{t} e^{\varepsilon s}\left(\int_{-\tau}^{0} V^{w_{i}^{\prime}}(\mathbf{x}(s+\theta), s+\theta) \mathrm{d} \eta_{i}(\theta)-e^{\varepsilon \tau} V^{w_{i}^{\prime}}(\mathbf{x}(s), s)\right) \mathrm{d} s \\
& \leq e^{\varepsilon \tau} \int_{-\tau}^{0} V^{w_{i}^{\prime}}(\mathbf{x}(s), s) \mathrm{d} s
\end{aligned}
$$

for $w_{1}^{\prime}=\beta_{2}, w_{2}^{\prime}=\beta_{1}, w_{3}^{\prime}=1$, respectively, and using Itô’s formula, we have

$$
\begin{aligned}
e^{\varepsilon t} V(\mathbf{x}(t), t)= & V(\mathbf{x}(0), 0)+\int_{0}^{t} e^{\varepsilon s}\left(\mathscr{L} V\left(\mathbf{x}_{s}, s\right)+\varepsilon V(\mathbf{x}(s), s)\right) \mathrm{d} s+M_{2}(t) \\
\leq & V(\mathbf{x}(0), 0)-\bar{R} \int_{0}^{t} e^{\varepsilon s} V(\mathbf{x}(s), s) \mathrm{d} s+k_{1} e^{\varepsilon \tau} \int_{-\tau}^{0} V^{\beta_{2}}(\mathbf{x}(s), s) \mathrm{d} s \\
& +k_{2} e^{\varepsilon \tau} \int_{-\tau}^{0} V^{\beta_{1}}(\mathbf{x}(s), s) \mathrm{d} s+k_{3} e^{\varepsilon \tau} \int_{-\tau}^{0} V(\mathbf{x}(s), s) \mathrm{d} s+M_{2}(t),
\end{aligned}
$$

where $M_{2}(t)=\int_{0}^{t} e^{\varepsilon s} V_{x}(\mathbf{x}(s), s) g\left(\mathbf{x}_{s}, s\right) \mathrm{d} B(s)$ is a local martingale with the initial value $M_{2}(0)=0$. Applying the nonnegative semi-martingale convergence theorem (see Lemma 2.5), we obtain that

$$
\limsup _{t \rightarrow \infty} e^{\varepsilon t} V(\mathbf{x}(t), t)<\infty \quad \text { a.s. }
$$

By the same technique as (16) in Theorem 3.1, letting $\varepsilon \rightarrow \bar{\varepsilon}$, we claim that

$$
\limsup _{t \rightarrow \infty} \frac{\log (V(\mathbf{x}(t), t))}{t} \leq-\bar{\varepsilon} \quad \text { a.s. }
$$

Next, we prove the other result. From (33), we get that

$$
\begin{aligned}
E e^{\varepsilon t} V(\mathbf{x}(t), t) \leq & E V(\mathbf{x}(0), 0)-\bar{R} E \int_{0}^{t} e^{\varepsilon s} V(\mathbf{x}(s), s) \mathrm{d} s+k_{1} e^{\varepsilon \tau} E \int_{-\tau}^{0} V^{\beta_{2}}(\mathbf{x}(s), s) \mathrm{d} s \\
& +k_{2} e^{\varepsilon \tau} E \int_{-\tau}^{0} V^{\beta_{1}}(\mathbf{x}(s), s) \mathrm{d} s+k_{3} e^{\varepsilon \tau} E \int_{-\tau}^{0} V(\mathbf{x}(s), s) \mathrm{d} s \\
\leq & c_{4},
\end{aligned}
$$


where $c_{4}=E V(\mathbf{x}(0), 0)+k_{1} e^{\varepsilon \tau} E \int_{-\tau}^{0} V^{\beta_{2}}(\mathbf{x}(s), s) \mathrm{d} s+k_{2} e^{\varepsilon \tau} E \int_{-\tau}^{0} V^{\beta_{1}}(\mathbf{x}(s), s) \mathrm{d} s+k_{3} e^{\varepsilon \tau} E \times$ $\int_{-\tau}^{0} V(\mathbf{x}(s), s) \mathrm{d} s$. This implies

$$
E V(\mathbf{x}(t), t) \leq c_{4} e^{-\varepsilon t}, \quad \forall t>0
$$

So, letting $\varepsilon \rightarrow \bar{\varepsilon}$ in the inequality above, we claim that

$$
\limsup _{t \rightarrow \infty} \frac{\log (E V(\mathbf{x}(t), t))}{t} \leq-\bar{\varepsilon} .
$$

\section{Examples}

In this section, we shall discuss some examples to illustrate our theory.

Example 1 Consider the scalar generalized stochastic logistic differential system of population extinction as follows:

$$
\mathrm{d} \mathbf{x}(t)=\mathbf{x}(t)(-2-\mathbf{x}(t)) \mathrm{d} t+\mathbf{x}(t)\left(1+\mathbf{x}^{\frac{1}{3}}(t)\right) \mathrm{d} B(t)
$$

with initial data $\mathbf{x}(0)>0, \mathbf{x}(0) \in C$, where $B(t)$ is a scalar Brownian motion.

Similar to the proof of Theorem 2.1 of [23], using the Lyapunov function $V(\mathbf{x})=\mathbf{x}-$ $1-\log (\mathbf{x})$, we claim that the solution of system (37) is not only positive but also will not explode to infinity at any finite time; and we compute that

$$
\begin{aligned}
& \mathbf{x}^{T} \mathbf{f}\left(\mathbf{x}_{t}, t\right) \leq-|\mathbf{x}(t)|^{3}-2|\mathbf{x}(t)|^{2} \\
& \left|\mathbf{g}\left(\mathbf{x}_{t}, t\right)\right| \leq|\mathbf{x}(t)|+|\mathbf{x}(t)|^{\frac{4}{3}}
\end{aligned}
$$

So, the parameters used in Assumption 2.2 are $\kappa=1, \bar{\kappa}=0, \hat{\kappa}=2, \underline{\kappa}=0, \gamma=1, \bar{\gamma}=0$, $\hat{\gamma}=1, \underline{\gamma}=0, n_{1}=2, n_{2}=4 / 3$. Through a simple computation we obtain that $L=\left(n_{1}-2 n_{2}+\right.$ 1) $\left(2 n_{2}-2\right)^{\frac{2 n_{2}-2}{n_{1}-2 n_{2}+1}}\left(n_{1}-1\right)^{\frac{1-n_{1}}{n_{1}-2 n_{2}+1}}=\frac{4}{27}, p_{0}=1+\left[\frac{(\gamma+\bar{\gamma})^{2}}{2(\kappa-\bar{\kappa})}+\frac{(\hat{\gamma}+\underline{\gamma})^{2}}{2(\hat{\kappa}-\underline{\kappa})-2 L(\kappa-\bar{\kappa})}\right]^{-1}=2.3$, and condition (4) is satisfied.

Through Theorem 3.1, we claim that the solution of system (37) is pth moment asymptotically stable for any $p \in(0,2.3)$, namely

$$
\lim _{t \rightarrow \infty} E|\mathbf{x}(t, \zeta)|^{p}=0
$$

Through Corollary 4.1, we claim that the solution of system (37) is almost surely exponentially stable and $p$ th moment exponentially stable, namely

$$
\begin{aligned}
& \limsup _{t \rightarrow \infty} \frac{\log (|\mathbf{x}(t, \zeta)|)}{t} \leq-\frac{\varepsilon_{p}^{\prime}}{p}, \quad p \in[2,2.3) \text { a.s., } \\
& \limsup _{t \rightarrow \infty} \frac{\log \left(E|\mathbf{x}(t, \zeta)|^{p}\right)}{t} \leq-\varepsilon_{p}^{\prime}, \quad p \in[2,2.3), \\
& \limsup _{t \rightarrow \infty} \frac{\log \left(E|\mathbf{x}(t, \zeta)|^{p}\right)}{t} \leq-\frac{p}{2} \varepsilon_{2}^{\prime}, \quad p \in(0,2),
\end{aligned}
$$

where $\varepsilon_{p}^{\prime}$ is a positive constant which only depends on $p$ but not on initial data $\mathbf{x}(0)$. Figure 1 shows a stochastic trajectory generated by the Euler scheme for time step $\triangle=10^{-5}$ for stochastic system $(37)$ on $[0,1]$ with initial data $\zeta=10$. 


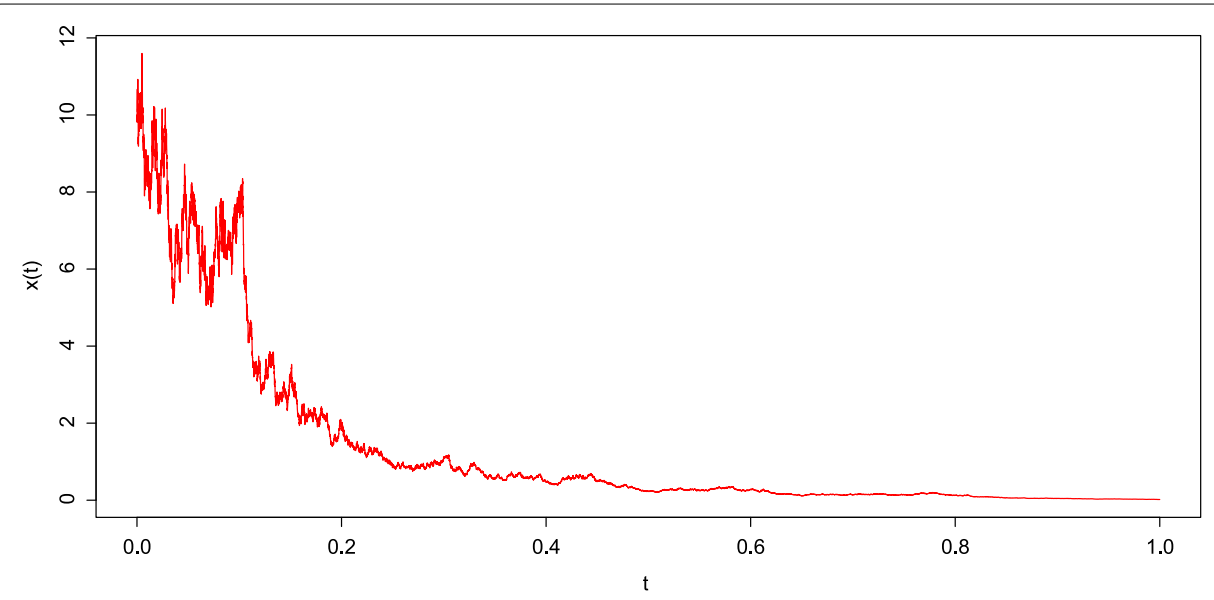

Figure 1 Generalized stochastic logistic differential system. The red curve shows a stochastic trajectory generated by the Euler scheme for time step $\Delta=10^{-5}$ for one-dimensional stochastic system (37) on $[0,1]$ with initial data $\zeta=10$ in Example 1.

Example 2 Let us consider the scalar SFDE as follows:

$$
\begin{aligned}
\mathrm{d} \mathbf{x}(t)= & \left(-4 \mathbf{x}^{5}(t)-6 \mathbf{x}(t)+2 D_{1}^{3}\left(\mathbf{x}_{t}\right)+2 D_{2}\left(\mathbf{x}_{t}\right)+\xi(t)\right) \mathrm{d} t+\frac{1}{2}\left(\mathbf{x}^{2}(t)+\mathbf{x}(t)\right. \\
& \left.+D_{3}^{2}\left(\mathbf{x}_{t}\right)+D_{4}\left(\mathbf{x}_{t}\right)+\xi(t)\right) \mathrm{d} B(t)
\end{aligned}
$$

with initial data $\{\mathbf{x}(\theta):-\tau \leq \theta \leq 0\}=\zeta \in C$, where $B(t)$ is a scalar Brownian motion. Moreover, $D_{i}$ are bounded linear operators from $C([-\tau, 0] ; R)$ to $R$ satisfying $\left|D_{i}\left(\mathbf{x}_{t}\right)\right| \leq$ $\int_{-\tau}^{0}|\mathbf{x}(t+\theta)| \mathrm{d} \eta_{i}(\theta)$, where $\eta_{i}(\cdot)$ are probability measures on $[-\tau, 0], i=1,2,3,4$.

(1) If the function $\xi(t)$ is defined by

$$
\xi(t)= \begin{cases}1-4^{n}|t-n|, & t \in\left[n-\frac{1}{4^{n}}, n+\frac{1}{4^{n}}\right], n=1,2,3, \ldots, \\ 0, & \text { others, }\end{cases}
$$

then it is easy to show that $\xi(t)$ is bounded, and $\int_{0}^{+\infty} \xi(t) \mathrm{d} t=1$. We compute that

$$
\begin{aligned}
\mathbf{x}^{T} \mathbf{f}\left(\mathbf{x}_{t}, t\right)= & -4|\mathbf{x}(t)|^{6}+2 \mathbf{x}(t)^{T} D_{1}^{3}\left(\mathbf{x}_{t}\right)-6|\mathbf{x}(t)|^{2}+2 \mathbf{x}(t)^{T} D_{2}\left(\mathbf{x}_{t}\right)+\mathbf{x}(t)^{T} \xi(t) \\
\leq & -4|\mathbf{x}(t)|^{6}+\int_{-\tau}^{0}|\mathbf{x}(t+\theta)|^{6} \mathrm{~d} \eta_{1}(\theta)-\frac{7}{2}|\mathbf{x}(t)|^{2} \\
& +\int_{-\tau}^{0}|\mathbf{x}(t+\theta)|^{2} \mathrm{~d} \eta_{2}(\theta)+\frac{1}{2} \xi^{2}(t), \\
\left|\mathbf{g}\left(\mathbf{x}_{t}, t\right)\right| \leq & \frac{1}{2}\left(|\mathbf{x}(t)|^{2}+|\mathbf{x}(t)|+\left|D_{3}\left(\mathbf{x}_{t}\right)\right|^{2}+\left|D_{4}\left(\mathbf{x}_{t}\right)\right|+\xi(t)\right) \\
\leq & \frac{1}{2}\left(|\mathbf{x}(t)|^{2}+\int_{-\tau}^{0}|\mathbf{x}(t+\theta)|^{2} \mathrm{~d} \eta_{3}(\theta)+|\mathbf{x}(t)|+\int_{-\tau}^{0}|\mathbf{x}(t+\theta)| \mathrm{d} \eta_{4}(\theta)+\xi(t)\right) .
\end{aligned}
$$

So, we obtain that $L=\left(n_{1}-2 n_{2}+1\right)\left(2 n_{2}-2\right)^{\frac{2 n_{2}-2}{n_{1}-2 n_{2}+1}}\left(n_{1}-1\right)^{\frac{1-n_{1}}{n_{1}-2 n_{2}+1}}=(5-4+1)(4-2)^{\frac{2}{2}}(5-$ $1)^{\frac{-4}{2}}=\frac{1}{4}, p_{0}=1+\left[\frac{(\gamma+\bar{\gamma})^{2}}{2(\kappa-\bar{\kappa})}+\frac{(\hat{\gamma}+\gamma)^{2}}{2(\hat{\kappa}-\kappa)-2 L(\kappa-\bar{\kappa})}\right]^{-1}=\frac{61}{19}>2$, and condition (4) becomes $\frac{(\gamma+\bar{\gamma})^{2}}{2(\kappa-\bar{\kappa})}+$ $\frac{(\hat{\gamma}+\underline{\gamma})^{2}}{2(\hat{\kappa}-\underline{\kappa})-2 L(\kappa-\bar{\kappa})}=\frac{1}{2(4-1)}+\frac{1}{5-\frac{3}{2}}=\frac{19}{42}<1,(\hat{\kappa}-\underline{\kappa})-L(\kappa-\bar{\kappa})=\frac{5}{2}-\frac{3}{4}>0$. 
Through Theorem 3.1, we claim that for any initial data $\zeta \in C$, the solution of system (38) is $p$ th moment asymptotically stable for any $p \in\left(0, \frac{61}{19}\right)$, namely

$$
\lim _{t \rightarrow \infty} E|\mathbf{x}(t, \zeta)|^{p}=0
$$

(2) If $\xi(t)=e^{-t}$, then it is also easy to show that $\xi(t)$ is bounded, and $\int_{0}^{+\infty} \xi(t) \mathrm{d} t=1$.

Through Theorem 3.1, we claim that for any initial data $\zeta \in C$, the solution of system (38) is $p$ th moment asymptotically stable for any $p \in\left(0, \frac{61}{19}\right)$, namely

$$
\lim _{t \rightarrow \infty} E|\mathbf{x}(t, \zeta)|^{p}=0
$$

Through Theorem 4.1, we claim that for any initial data $\zeta \in C$, the solution of system (38) is almost surely exponentially stable and $p$ th moment exponentially stable, namely

$$
\begin{aligned}
& \limsup _{t \rightarrow \infty} \frac{\log (|\mathbf{x}(t, \zeta)|)}{t} \leq-\frac{\varepsilon_{p}}{p}, \quad p \in\left[2, \frac{61}{19}\right) \text { a.s., } \\
& \limsup _{t \rightarrow \infty} \frac{\log \left(E|\mathbf{x}(t, \zeta)|^{p}\right)}{t} \leq-\varepsilon_{p}, \quad p \in\left[2, \frac{61}{19}\right) \\
& \limsup _{t \rightarrow \infty} \frac{\log \left(E|\mathbf{x}(t, \zeta)|^{p}\right)}{t} \leq-\frac{p}{2} \varepsilon_{2}, \quad p \in(0,2),
\end{aligned}
$$

where $\varepsilon_{p}$ is defined by

$$
\begin{gathered}
\varepsilon_{p}=\sup \left\{\varepsilon>0: \frac{1+e^{\varepsilon \tau}}{4\left(4-e^{\varepsilon \tau}\right)}+\frac{1+e^{\varepsilon \tau}}{10-\frac{4}{p} \varepsilon-3 e^{\varepsilon \tau}}<\frac{1}{p-1},\right. \\
\left.10-\frac{4}{p} \varepsilon-3 e^{\varepsilon \tau}>0,4-e^{\varepsilon \tau}>0, \varepsilon<p\right\}
\end{gathered}
$$

\section{Appendix}

Proof of Lemma 3.1 For any given initial data $\zeta \in C$, by [1], Assumption 2.1 and conditions $\mathbf{f}(0, t)=0$ and $\mathbf{g}(0, t)=0$ guarantee a unique maximal local solution $\mathbf{x}(t, \zeta)$ to system (1) on $t \in\left[-\tau, \sigma_{\infty}\right)$, where $\sigma_{\infty}$ is the explosion time. Let $k_{0}>0$ be sufficiently large satisfying $\|\zeta\|<k_{0}$. For each integer $k \geq k_{0}$, define the stopping time $\tau_{k}=\inf \left\{t \in\left[0, \sigma_{\infty}\right):|x(t)| \geq k\right\}$. Obviously, $\tau_{k}$ is increasing as $k \rightarrow \infty$. Let $\tau_{\infty}=\lim _{t \rightarrow \infty} \tau_{k}$, so $\tau_{\infty} \leq \sigma_{\infty}$ a.s. If we can obtain that $\tau_{\infty}=\infty$ a.s., then $\sigma_{\infty}=\infty$ a.s. For the sake of simplicity, write $\mathbf{x}(t)=\mathbf{x}(t, \zeta)$. Using Itô's formula to $V(\mathbf{x}, t)=|\mathbf{x}(t)|^{2}$, we obtain

$$
\begin{aligned}
\mathscr{L} V(\mathbf{x}, t)= & 2 \mathbf{x}^{T}(t) \mathbf{f}\left(\mathbf{x}_{t}, t\right)+\left|\mathbf{g}\left(\mathbf{x}_{t}, t\right)\right|^{2} \\
\leq & 2\left(-\kappa|\mathbf{x}(t)|^{n_{1}+1}+\bar{\kappa} \int_{-\tau}^{0}|\mathbf{x}(t+\theta)|^{n_{1}+1} \mathrm{~d} \eta_{1}(\theta)-\hat{\kappa}|\mathbf{x}(t)|^{2}\right. \\
& \left.+\underline{\kappa} \int_{-\tau}^{0}|\mathbf{x}(t+\theta)|^{2} \mathrm{~d} \eta_{2}(\theta)+\xi_{1}(t)\right) \\
& +5\left(\gamma^{2}|\mathbf{x}(t)|^{2 n_{2}}+\bar{\gamma}^{2} \int_{-\tau}^{0}|\mathbf{x}(t+\theta)|^{2 n_{2}} \mathrm{~d} \eta_{3}(\theta)\right. \\
& \left.+\hat{\gamma}^{2}|\mathbf{x}(t)|^{2}+\underline{\gamma}^{2} \int_{-\tau}^{0}|\mathbf{x}(t+\theta)|^{2} \mathrm{~d} \eta_{4}(\theta)+\xi_{2}^{2}(t)\right)
\end{aligned}
$$




$$
\begin{aligned}
\leq & -2(\kappa-\bar{\kappa})|\mathbf{x}(t)|^{n_{1}+1}+5\left(\gamma^{2}+\bar{\gamma}^{2}\right)|\mathbf{x}(t)|^{2 n_{2}}+\left(5\left(\hat{\gamma}^{2}+\underline{\gamma}^{2}\right)-2(\hat{\kappa}-\underline{\kappa})\right)|\mathbf{x}(t)|^{2} \\
& +2 \bar{\kappa} \tilde{J}_{1}+2 \underline{\kappa}_{2}+5 \bar{\gamma}^{2} \tilde{J}_{3}+5 \underline{\gamma}^{2} \tilde{J}_{4}+2 \xi_{1}(t)+5 \xi_{2}^{2}(t),
\end{aligned}
$$

where $\tilde{J}_{1}=\int_{-\tau}^{0}|\mathbf{x}(t+\theta)|^{n_{1}+1} \mathrm{~d} \eta_{1}(\theta)-|\mathbf{x}(t)|^{n_{1}+1}, \tilde{J}_{2}=\int_{-\tau}^{0}|\mathbf{x}(t+\theta)|^{2} \mathrm{~d} \eta_{2}(\theta)-|\mathbf{x}(t)|^{2}, \tilde{J}_{3}=$ $\int_{-\tau}^{0}|\mathbf{x}(t+\theta)|^{2 n_{2}} \mathrm{~d} \eta_{3}(\theta)-|\mathbf{x}(t)|^{2 n_{2}}, \tilde{J}_{4}=\int_{-\tau}^{0}|\mathbf{x}(t+\theta)|^{2} \mathrm{~d} \eta_{4}(\theta)-|\mathbf{x}(t)|^{2}$.

Noting $\kappa>\bar{\kappa}, n_{1}+1>2 n_{2} \geq 2$ and $|\mathbf{x}(t)| \geq 0$ for any $t \geq 0$, by Lemma 2.4, $R_{1}(|\mathbf{x}(t)|)=$ $-2(\kappa-\bar{\kappa})|\mathbf{x}(t)|^{n_{1}+1}+5\left(\gamma^{2}+\bar{\gamma}^{2}\right)|\mathbf{x}(t)|^{2 n_{2}}+\left(5\left(\hat{\gamma}^{2}+\underline{\gamma}^{2}\right)-2(\hat{\kappa}-\underline{\kappa})\right)|\mathbf{x}(t)|^{2}$, as a function of $|\mathbf{x}(t)|$, has a positive upper-boundedness, i.e., there is a positive constant $\tilde{R}_{1}$ such that

$$
\begin{aligned}
R_{1}(|\mathbf{x}(t)|) & =-2(\kappa-\bar{\kappa})|\mathbf{x}(t)|^{n_{1}+1}+5\left(\gamma^{2}+\bar{\gamma}^{2}\right)|\mathbf{x}(t)|^{2 n_{2}}+\left(5\left(\hat{\gamma}^{2}+\underline{\gamma}^{2}\right)-2(\hat{\kappa}-\underline{\kappa})\right)|\mathbf{x}(t)|^{2} \\
& \leq \tilde{R}_{1} .
\end{aligned}
$$

(This technique has been used by many researchers, for example, [12].)

From Lemma 2.2, we have $\int_{0}^{+\infty}\left(5 \xi_{2}^{2}(s)+2 \xi_{1}(s)\right) \mathrm{d} s<\infty$. In view of the fact that

$$
\int_{0}^{t \wedge \tau_{k}} \tilde{J}_{i} \mathrm{~d} s=\int_{0}^{t \wedge \tau_{k}}\left(\int_{-\tau}^{0}|\mathbf{x}(s+\theta)|^{w_{i}^{\prime}} \mathrm{d} \eta_{i}(\theta)-|\mathbf{x}(s)|^{w_{i}^{\prime}}\right) \mathrm{d} s \leq \int_{-\tau}^{0}|\mathbf{x}(s)|^{w_{i}^{\prime}} \mathrm{d} s
$$

for $w_{1}^{\prime}=n_{1}+1, w_{3}^{\prime}=2 n_{2}, w_{2}^{\prime}=w_{4}^{\prime}=2$, we have that, for $t \geq 0$,

$$
\begin{aligned}
E\left|\mathbf{x}\left(t \wedge \tau_{k}\right)\right|^{2}= & E|\mathbf{x}(0)|^{2}+E \int_{0}^{t \wedge \tau_{k}}\left[2 \mathbf{x}^{T}(s) \mathbf{f}\left(\mathbf{x}_{s}, s\right)+\left|\mathbf{g}\left(\mathbf{x}_{s}, s\right)\right|\right]^{2} \mathrm{~d} s \\
\leq & E|\mathbf{x}(0)|^{2}+E \int_{0}^{t \wedge \tau_{k}}\left[\tilde{R}_{1}+2 \tilde{\kappa} \tilde{J}_{1}+2 \underline{\kappa} \tilde{J}_{2}+5 \bar{\gamma}^{2} \tilde{J}_{3}\right. \\
& \left.+5 \underline{\gamma^{2}} \tilde{J}_{4}+2 \xi_{1}(s)+5 \xi_{2}^{2}(s)\right] \mathrm{d} s \\
\leq & E|\mathbf{x}(0)|^{2}+\tilde{R}_{1} E\left(t \wedge \tau_{k}\right)+2 \bar{\kappa} \int_{-\tau}^{0}|\mathbf{x}(s)|^{n_{1}+1} \mathrm{~d} s+2 \underline{\kappa} \int_{-\tau}^{0}|\mathbf{x}(s)|^{2} \mathrm{~d} s \\
& +5 \bar{\gamma}^{2} \int_{-\tau}^{0}|\mathbf{x}(s)|^{2 n_{2}} \mathrm{~d} s+5 \underline{\gamma}^{2} \int_{-\tau}^{0}|\mathbf{x}(s)|^{2} \mathrm{~d} s+\int_{0}^{\infty}\left[2 \xi_{1}(s)+5 \xi_{2}^{2}(s)\right] \mathrm{d} s \\
\leq & \bar{R}_{1}+\tilde{R}_{1} t
\end{aligned}
$$

where $\bar{R}_{1}=E|\mathbf{x}(0)|^{2}+2 \bar{\kappa} \int_{-\tau}^{0}|\mathbf{x}(s)|^{n_{1}+1} \mathrm{~d} s+2 \underline{\kappa} \int_{-\tau}^{0}|\mathbf{x}(s)|^{2} \mathrm{~d} s+5 \bar{\gamma}^{2} \int_{-\tau}^{0}|\mathbf{x}(s)|^{2 n_{2}} \mathrm{~d} s+5 \underline{\gamma}^{2} \times$ $\int_{-\tau}^{0}|\mathbf{x}(s)|^{2} \mathrm{~d} s+\int_{0}^{\infty}\left[2 \xi_{1}(s)+5 \xi_{2}^{2}(s)\right] \mathrm{d} s$. Noting that

$$
E\left|\mathbf{x}\left(t \wedge \tau_{k}\right)\right|^{2} \geq E\left(\left|\mathbf{x}\left(t \wedge \tau_{k}\right)\right|^{2} I_{\left\{\tau_{k} \leq t\right\}}\right) \geq k^{2} P\left\{\tau_{k} \leq t\right\}
$$

we get that

$$
P\left\{\tau_{\infty} \leq t\right\}=\lim _{k \rightarrow \infty} P\left\{\tau_{k} \leq t\right\} \leq \lim _{k \rightarrow \infty} \frac{\bar{R}_{1}+\tilde{R}_{1} t}{k^{2}}=0
$$

Since $t$ is arbitrary, we must have that $\tau_{\infty}=\infty$ a.s. and this completes the proof.

Proof of Lemma 3.2 Since $\kappa>\bar{\kappa}$, the existence and uniqueness of the solution follows from Lemma 3.1, and there exists at least a sufficiently small positive constant $\varepsilon$ satisfying $\kappa>$ 
$\bar{\kappa} e^{\varepsilon \tau}$. So, by the continuity, define $\varepsilon^{\prime \prime}=\sup \left\{\varepsilon>0: \kappa>\bar{\kappa} e^{\varepsilon \tau}\right\}$. For the sake of simplicity, write $\mathbf{x}(t)=\mathbf{x}(t, \zeta), \mathbf{x}_{t}=\mathbf{x}_{t}^{\zeta}$. For any $p \geq 2$, applying Itô's formula to $V(\mathbf{x}, t)=e^{\varepsilon t}|\mathbf{x}(t)|^{p}, \varepsilon \in\left(0, \varepsilon^{\prime \prime}\right]$, we arrive at

$$
\begin{aligned}
\mathscr{L} V(\mathbf{x}, t)= & e^{\varepsilon t}\left(\mathscr{L}|\mathbf{x}(t)|^{p}+\varepsilon|\mathbf{x}(t)|^{p}\right) \\
\leq & e^{\varepsilon t}\left[\frac{p}{2}|\mathbf{x}(t)|^{p-2}\left(2 \mathbf{x}^{T}(r) \mathbf{f}\left(\mathbf{x}_{r}, r\right)+(p-1)\left|\mathbf{g}\left(\mathbf{x}_{r}, r\right)\right|^{2}\right)+\varepsilon|\mathbf{x}(t)|^{p}\right] \\
\leq & e^{\varepsilon t} \frac{p}{2}|\mathbf{x}(t)|^{p-2}\left[2 \left(-\kappa|\mathbf{x}(t)|^{n_{1}+1}+\bar{\kappa} \int_{-\tau}^{0}|\mathbf{x}(t+\theta)|^{n_{1}+1} \mathrm{~d} \eta_{1}(\theta)-|\mathbf{x}(t)|^{2}\right.\right. \\
& \left.+\underline{\kappa} \int_{-\tau}^{0}|\mathbf{x}(t+\theta)|^{2} \mathrm{~d} \eta_{2}(\theta)+\xi_{1}(t)\right)+5(p-1)\left(\gamma^{2}|\mathbf{x}(t)|^{2 n_{2}}\right. \\
& +\bar{\gamma}^{2} \int_{-\tau}^{0}|\mathbf{x}(t+\theta)|^{2 n_{2}} \mathrm{~d} \eta_{3}(\theta)+\hat{\gamma}^{2}|\mathbf{x}(t)|^{2} \\
& \left.\left.+\underline{\gamma}^{2} \int_{-\tau}^{0}|\mathbf{x}(t+\theta)|^{2} \mathrm{~d} \eta_{4}(\theta)+\xi_{2}^{2}(t)\right)+\frac{2}{p} \varepsilon|\mathbf{x}(t)|^{2}\right] \\
\leq & \frac{p}{2} e^{\varepsilon t}\left[-2\left(\kappa-\bar{\kappa} e^{\varepsilon \tau}\right)|\mathbf{x}(t)|^{p+n_{1}-1}+o\left(|\mathbf{x}(t)|^{p+n_{1}-1}\right)\right]+2 \underline{\kappa} e^{\varepsilon t} J_{2} \\
& +5(p-1) \underline{\gamma}^{2} e^{\varepsilon t} J_{4}+\frac{p}{2} 5(p-1) \bar{\gamma}^{2} \frac{2 n_{2}}{p+2 n_{2}-2} e^{\varepsilon t} J_{3}+p \bar{\kappa} \frac{n_{1}+1}{p+n_{1}-1} e^{\varepsilon t} J_{1} \\
& +2 e^{\varepsilon t} \xi_{1}^{\frac{p}{2}}(t)+5(p-1) e^{\varepsilon t} \xi_{2}^{p}(t),
\end{aligned}
$$

where $J_{1}=\int_{-\tau}^{0}|\mathbf{x}(t+\theta)|^{p+n_{1}-1} \mathrm{~d} \eta_{1}(\theta)-e^{\varepsilon \tau}|\mathbf{x}(t)|^{p+n_{1}-1}, J_{2}=\int_{-\tau}^{0}|\mathbf{x}(t+\theta)|^{p} \mathrm{~d} \eta_{2}(\theta)-e^{\varepsilon \tau}|\mathbf{x}(t)|^{p}$, $J_{3}=\int_{-\tau}^{0}|\mathbf{x}(t+\theta)|^{p+2 n_{2}-2} \mathrm{~d} \eta_{3}(\theta)-e^{\varepsilon \tau}|\mathbf{x}(t)|^{p+2 n_{2}-2}, J_{4}=\int_{-\tau}^{0}|\mathbf{x}(t+\theta)|^{p} \mathrm{~d} \eta_{4}(\theta)-e^{\varepsilon \tau}|\mathbf{x}(t)|^{p}$.

Noting that $\kappa>\bar{\kappa} e^{\varepsilon \tau}$ and $|\mathbf{x}(t)| \geq 0$ for any $t \geq 0$, by Lemma 2.4 and the same technique as (39), $\bar{G}(|\mathbf{x}(t)|)=-2\left(\kappa-\bar{\kappa} e^{\varepsilon \tau}\right)|\mathbf{x}(t)|^{p+n_{1}-1}+o\left(|\mathbf{x}(t)|^{p+n_{1}-1}\right)$, as a function of $|\mathbf{x}(t)|$, has a positive upper-boundedness, i.e., there is a positive constant $\underline{Q}$ such that

$$
\bar{G}(|\mathbf{x}(t)|)=-2\left(\kappa-\bar{\kappa} e^{\varepsilon \tau}\right)|\mathbf{x}(t)|^{p+n_{1}-1}+o\left(|\mathbf{x}(t)|^{p+n_{1}-1}\right) \leq \underline{Q} .
$$

In view of the fact that

$$
\int_{0}^{t} e^{\varepsilon s} J_{i} \mathrm{~d} s=\int_{0}^{t} e^{\varepsilon s}\left(\int_{-\tau}^{0}|\mathbf{x}(s+\theta)|^{w_{i}} \mathrm{~d} \eta_{i}(\theta)-e^{\varepsilon \tau}|\mathbf{x}(s)|^{w_{i}}\right) \mathrm{d} s \leq e^{\varepsilon \tau} \int_{-\tau}^{0}|\mathbf{x}(s)|^{w_{i}} \mathrm{~d} s
$$

for $w_{1}=p+n_{1}-1, w_{3}=p+2 n_{2}-2, w_{2}=w_{4}=p$, we get that, for $t \geq 0$,

$$
\begin{aligned}
E e^{\varepsilon t}|\mathbf{x}(t)|^{p} \leq & E|\mathbf{x}(0)|^{p}+\frac{p}{2} \underline{Q} \int_{0}^{t} e^{\varepsilon s} \mathrm{~d} s+p \bar{\kappa} \frac{n_{1}+1}{p+n_{1}-1} e^{\varepsilon \tau} \int_{-\tau}^{0}|\mathbf{x}(s)|^{p+n_{1}-1} \mathrm{~d} s \\
& +2 \underline{\kappa} e^{\varepsilon \tau} \int_{-\tau}^{0}|\mathbf{x}(s)|^{p} \mathrm{~d} s+\frac{p}{2} 5(p-1) \bar{\gamma}^{2} \frac{2 n_{2}}{p+2 n_{2}-2} e^{\varepsilon \tau} \int_{-\tau}^{0}|\mathbf{x}(s)|^{p+2 n_{2}-2} \mathrm{~d} s \\
& +5(p-1) \underline{\gamma}^{2} e^{\varepsilon \tau} \int_{-\tau}^{0}|\mathbf{x}(s)|^{p} \mathrm{~d} s+\int_{0}^{t} e^{\varepsilon s}\left[2 \xi_{1}^{\frac{p}{2}}(s)+5(p-1) \xi_{2}^{p}(s)\right] \mathrm{d} s .
\end{aligned}
$$

By virtue of the boundedness of $\xi_{1}(t), \xi_{2}(t)$, there is a constant $\Psi>0$ such that $\xi_{1}(t) \vee \xi_{2}(t) \leq$ $\Psi$, which implies that

$$
E e^{\varepsilon t}|\mathbf{x}(t)|^{p} \leq c+\left[2 \Psi^{\frac{p}{2}}+5(p-1) \Psi^{p}+\frac{p}{2} \underline{Q}\right] \int_{0}^{t} e^{\varepsilon s} \mathrm{~d} s,
$$


where $c=p \bar{\kappa} \frac{n_{1}+1}{p+n_{1}-1} e^{\varepsilon \tau} \int_{-\tau}^{0}|\mathbf{x}(s)|^{p+n_{1}-1} \mathrm{~d} s+\frac{p}{2} 5(p-1) \bar{\gamma}^{2} \frac{2 n_{2}}{p+2 n_{2}-2} e^{\varepsilon \tau} \int_{-\tau}^{0}|\mathbf{x}(s)|^{p+2 n_{2}-2} \mathrm{~d} s+2 \underline{\kappa} e^{\varepsilon \tau} \times$ $\int_{-\tau}^{0}|\mathbf{x}(s)|^{p} \mathrm{~d} s+5(p-1) \underline{\gamma}^{2} e^{\varepsilon \tau} \int_{-\tau}^{0}|\mathbf{x}(s)|^{p} \mathrm{~d} s+E|\mathbf{x}(0)|^{p}$. This implies

$$
E|\mathbf{x}(t)|^{p} \leq c e^{-\varepsilon t}+\frac{2 \Psi^{\frac{p}{2}}+5(p-1) \Psi^{p}+\frac{p}{2} \underline{Q}}{\varepsilon}\left(1-e^{-\varepsilon t}\right) .
$$

From the boundedness of initial data $\zeta \in C$, we claim that for any $p \geq 2$, there exists a constant $M_{p}>0$ such that $\sup _{-\tau \leq t<+\infty} E|\mathbf{x}(t, \zeta)|^{p} \leq M_{p}$. When $p \in(0,2)$, using Hölder's inequality, we claim that

$$
\sup _{-\tau \leq t<\infty} E|\mathbf{x}(t)|^{p} \leq\left(\sup _{-\tau \leq t<\infty} E|\mathbf{x}(t)|^{2}\right)^{\frac{p}{2}} \leq M_{2}^{\frac{p}{2}} .
$$

\section{Competing interests}

The authors declare that there is no conflict of interests regarding the publication of this paper.

\section{Authors' contributions}

All authors contributed equally and significantly in writing this paper. All authors read and approved the final manuscript.

\section{Author details}

${ }^{1}$ College of Applied Sciences, Beijing University of Technology, 100 Pingleyuan, Chaoyang District, Beijing, 100124 P.R. China. ${ }^{2}$ College of Sciences, Hebei United University, 46 Xinhua Road, Tangshan, Hebei 063009, P.R. China.

\section{Acknowledgements}

The authors would like to thank the anonymous referees for their useful and valuable suggestions. This project was supported by the National Science Foundation of China (No. 11171010) and Tangshan Science and Technology Bureau Program of Hebei Province (No. $13130214 z$ and No. 13130203z)

\section{Received: 6 August 2014 Accepted: 14 November 2014 Published: 02 Dec 2014}

\section{References}

1. Mao, X: Stochastic Differential Equations and Applications. Horwood, Chichester (1997)

2. Mao, X, Yuan, C: Stochastic Differential Equations with Markovian Switching. Imperial College Press, London (2006)

3. Huang, L, Deng, F: Razumikhin-type theorems on stability of stochastic retarded systems. Int. J. Syst. Sci. 40, 73-80 (2009)

4. Liao, X, Mao, X: Exponential stability of stochastic delay interval systems. Syst. Control Lett. 40, 171-181 (2000)

5. Liu, L, Shen, Y, Jiang, F: Delay-dependent exponential stability of stochastic delay differential system whose coefficients obey the polynomial growth condition. Int. J. Syst. Sci. 43, 1664-1672 (2012)

6. Luo, Q, Mao, X, Shen, Y: Generalised theory on asymptotic stability and boundedness of stochastic functional differential equations. Automatica 47, 2075-2081 (2011)

7. Mao, X: Robustness of exponential stability of stochastic differential delay equations. IEEE Trans. Autom. Control 41, 442-447 (1996)

8. Mao, X: Razumikhin-type theorems on exponential stability of stochastic functional differential equations. Stoch. Process. Appl. 65, 233-250 (1996)

9. Mao, X, Koroleva, N, Rodkina, A: Robust stability of uncertain stochastic differential delay equations. Syst. Control Lett. 35, 325-336 (1998)

10. Mao, X: LaSalle-type theorems for stochastic differential delay equations. J. Math. Anal. Appl. 236, 350-369 (1999)

11. Pan, L, Cao, J: Exponential stability of impulsive stochastic functional differential equations. J. Math. Anal. Appl. 382, 672-685 (2011)

12. Wu, F, Hu, S: Stochastic suppression and stabilization of delay differential systems. Int. J. Robust Nonlinear Control 21, 488-500 (2011)

13. Yang, R, Shi, P, Gao, H: New delay-dependent stability criterion for stochastic systems with time delays. IET Control Theory Appl. 2, 966-973 (2008)

14. Appleby, JAD, Reynolds, DW: Decay rates of solutions of linear stochastic Volterra equations. Electron. J. Probab. 13, 922-943 (2008)

15. Appleby, JAD, Rodkina, A: Stability of nonlinear stochastic Volterra difference equations with respect to a fading perturbation. Int. J. Differ. Equ. 4, 165-184 (2009)

16. Shen, Y, Luo, Q, Mao, X: The improved LaSalle-type theorems for stochastic functional differential equations. J. Math Anal. Appl. 318, 134-154 (2006)

17. Mao, X: Numerical solutions of stochastic functional differential equations. LMS J Comput. Math 6, 141-161 (2003)

18. Popov, V: Hyperstability of Control System. Springer, Berlin (1973)

19. Lipster, RS, Shiryayev, AN: Theory of Martingales. Kluwer Academic, Dordrecht (1989)

20. Arnold, L: Stochastic Differential Equations: Theory and Applications. Wiley, New York (1972) 
21. Fang, S, Zhang, T: A study of a class of stochastic differential equations with non-Lipschitzian coefficients. Probab. Theory Relat. Fields 132, 356-390 (2005)

22. Xue, M, Zhou, S, Hu, S: Stability of nonlinear neutral stochastic functional differential equations. J. Appl. Math. 2010, Article ID 425762 (2010)

23. Ji, C, Jiang, D, Shi, N, O'Regan, D: Existence, uniqueness, stochastic persistence and global stability of positive solutions of the logistic equation with random perturbation. Math. Methods Appl. Sci. 30, 77-89 (2006)

10.1186/1687-1847-2014-302

Cite this article as: Feng and Li: The $p$ th moment asymptotic stability and exponential stability of stochastic

functional differential equations with polynomial growth condition. Advances in Difference Equations 2014, 2014:302

Submit your manuscript to a SpringerOpen ${ }^{\odot}$ journal and benefit from:

- Convenient online submission

Rigorous peer review

- Immediate publication on acceptance

- Open access: articles freely available online

- High visibility within the field

- Retaining the copyright to your article

Submit your next manuscript at $\gg$ springeropen.com 